\title{
Effects of Small-Scale Dead Wood Additions on Beetles in Southeastern U.S. Pine Forests
}

Kier D. Klepzig ${ }^{1, *}$, Michael L. Ferro ${ }^{2}$, Michael D. Ulyshen ${ }^{3}$, Matthew L. Gimmel ${ }^{4}$, Jolie B. Mahfouz ${ }^{5}$, Allan E. Tiarks ${ }^{5}$ and Chris E. Carlton ${ }^{2}$

1 USDA Forest Service, Southern Research Station, 200 WT Weaver Blvd, Asheville, NC 28804, USA

2 Department of Entomology, Louisiana State University, 404 Life Sciences Building, Baton Rouge, LA 70803-1710, USA; E-Mails: spongymesophyll@gmail.com (M.L.F.); ccarlt@1su.edu (C.E.C.)

3 USDA Forest Service, Southern Research Station, 201 Lincoln Green, Starkville, MS 39759, USA; E-Mail: mulyshen@fs.fed.us

4 Department of Ecology \& Evolutionary Biology, University of Kansas, Lawrence, KS 66045, USA; E-Mail: phalacrid@gmail.com

5 USDA Forest Service, Southern Research Station, 2500 Shreveport Hwy, Pineville, LA 71360, USA; E-Mails: joliemahfouz@msn.com (J.B.M.); aetiarks@att.net (A.E.T.)

* Author to whom correspondence should be addressed; E-Mail: kklepzig@fs.fed.us; Tel.: +1-828-257-4307; Fax: +1-828-257-4313.

Received: 19 June 2012; in revised form: 14 July 2012 / Accepted: 25 July 2012 /

Published: 15 August 2012

\begin{abstract}
Pitfall traps were used to sample beetles (Coleoptera) in plots with or without inputs of dead loblolly pine (Pinus taeda L.) wood at four locations (Louisiana, Mississippi, North Carolina and Texas) on the coastal plain of the southeastern United States. The plots were established in 1998 and sampling took place in 1998, 1999, and 2002 (only 1998 for North Carolina). Overall, beetles were more species rich, abundant and diverse in dead wood addition plots than in reference plots. While these differences were greatest in 1998 and lessened thereafter, they were not found to be significant in 1998 due largely to interactions between location and treatment. Specifically, the results from North Carolina were inconsistent with those from the other three locations. When these data were excluded from the analyses, the differences in overall beetle richness for 1998 became statistically significant. Beetle diversity was significantly higher in the dead wood plots in 1999 but by 2002 there were no differences between dead wood added and control plots. The positive influence of dead wood additions on the beetle community can be largely attributed to the saproxylic fauna (species dependent on dead wood), which, when analyzed separately,
\end{abstract}


were significantly more species rich and diverse in dead wood plots in 1998 and 1999. Ground beetles (Carabidae) and other species, by contrast, were not significantly affected. These results suggest manipulations of dead wood in pine forests have variable effects on beetles according to life history characteristics.

Keywords: arthropods; biodiversity; coarse woody debris; epigeic; insects; slash

\section{Introduction}

Logs and other woody debris on the forest floor are an essential habitat for a wide range of saproxylic arthropods [1-3], which are directly or indirectly dependent on dead and dying wood [4]. These include not only phloem and wood feeders but also their predators and species associated with wood-decaying fungi. Many non-saproxylic ground-dwelling arthropods benefit from dead wood as well. For instance, a number of studies have shown litter-dwelling arthropods and other invertebrates to be more numerous immediately next to dead wood than short distances away from it [5-17]. These results can be attributed variously to dead wood providing a relatively stable source of moisture [18-20], shelter from predators or adverse weather conditions, and an abundance of prey or other food items.

With demand for forest products continuing to rise (especially now with a growing interest in biofuel production, see [21,22]), understanding the role of dead wood in maintaining biodiversity, tree productivity and long-term forest sustainability is becoming more important in forest management. Of particular value are studies investigating the effects of dead wood manipulations on these interests. Several manipulative studies have demonstrated the positive relationship between dead wood and ground-dwelling arthropod diversity [23-25], but the question is far from resolved and has received little attention in many forest types.

The forests of the southeastern United States are among the most productive in North America with loblolly pine (Pinus taeda L.) being the single most important timber species in the region. One of the few efforts to measure arthropod response to dead wood manipulations made in loblolly pine plantations began on the Savannah River Site in South Carolina in 1996. In the first phase of that study, plots in which all dead wood was removed annually were compared to reference plots from which no dead wood was removed. After pitfall-sampling ground-dwelling arthropods in those plots for five years, Hanula et al. [26] found no differences in overall abundance or morphospecies richness. Overall arthropod diversity and evenness were significantly lower in removal plots than in reference plots, however, and several families differed in abundance between the two treatments. These differences were observed only in the first two full years of sampling, however. In the second phase of the study, beginning in 2001, arthropods were sampled in these same plots for four more years. Two new treatments were added in which sampling also took place; one involving major inputs of logs to the forest floor and the other involving major inputs of dead standing trees. Contrary to expectations, but largely consistent with the first phase of the study, there were no differences in total ground-dwelling arthropod abundance, richness, diversity or composition among treatments [27]. Only ground beetles (family Carabidae) exhibited a significant association with dead wood. 
During 1998, a study was installed on four widely-separated USDA Long Term Soil Productivity (LTSP) sites in the Southeastern United States to better understand the importance of dead wood to nutrient cycling and biodiversity in pine forests. The responses of beetles, the most diverse arthropods on the forest floor, are reported here.

\section{Materials and Methods}

This study took place at the following four LTSP sites: Croatan National Forest, Craven Country, NC, USA; DeSoto National Forest, Jones Country, MS, USA; Kisatchie National Forest, Rapides Parish, LA, USA; Davy Crockett National Forest, Trinity Country, TX, USA. The forests were dominated by 50-60 yr pine [loblolly in LA and NC, slash (P. elliottii Engelm.) in MS, and loblolly and shortleaf (P. echinata Mill.) in TX] with an understory consisting of oak (Quercus sp.), hickory (Carya sp.), and sweetgum (Liquidambar styraciflua L.). For this experiment, the coarse woody debris was added to remnant mature pine stand, adjacent to the LTSP treatment plots. At each site, three of the mature stand plots were used as controls and contained only the existing woody debris already present or that which fell during the study. A $30 \times 45 \mathrm{~m}$ rectangular area at each site was divided into six $15 \times$ $15 \mathrm{~m}$ plots. The dead wood added to all of the plots came from the same 43-year-old loblolly pine stand in the Palustris Experimental Forest, Louisiana. Sections of wood were removed from the main trunk (i.e., "logs", 30-35 cm dbh, $1 \mathrm{~m}$ long), limbs (2.5-10 cm mid-length diam, $0.5 \mathrm{~m}$ long), and twigs ( $0.5-1.5 \mathrm{~cm}$ diam, $0.25 \mathrm{~m}$ long) of loblolly pine trees felled for this purpose. Ten logs, 8 limbs, and 24 twigs were distributed across the three randomly selected dead wood plots at each site in April and May 1998. At each given site, the material was added all at once, on one date during the time frame mentioned above. At all sites, the amount of existing coarse woody debris and the vegetation on the ground were roughly the same. Our additions of material impacted the sites in a very similar manner, adding the above material to the baseline of that already onsite.

One pitfall trap (modified from [28]) was installed in each plot to sample ground-dwelling beetles. At each site, the trap was placed arbitrarily within the plot, in the immediate area of the added dead wood. Each trap consisted of a 15-cm-diameter plastic funnel fastened beneath a hole in the center of a $30 \times 30 \mathrm{~cm}$ section of $\sim 1.9 \mathrm{~cm}$-thick plywood with bevelled edges. This assembly was positioned over a 2-liter plastic container, half-filled with a 1:1 mixture of propylene glycol and 95\% ethanol, buried at ground level. Samples were collected approximately biweekly from May through September at all locations in 1998 and at all locations except for North Carolina in 1999. Additional samples were collected in all locations except for North Carolina in April and May of 2002.

Specimens not identified below family level (117 individuals were only identified to family level) were excluded from the dataset. After pooling all sampling periods for a given year, analyses of variance (ANOVA) were carried out for each year separately (due to differences in sampling intensity) to determine whether beetle abundance $(\log (x+1)$-transformed to achieve normality), richness, or Shannon's diversity varied between treatments. Site location (= "State") was included in the model as a blocking variable and was treated as a random effect. To provide additional information, separate analyses were performed on the following three groups: saproxylic (species directly or indirectly dependent on dead or dying wood), non-saproxylic ground beetles, and other species. These 
designations were based on familiarity with the taxa and information available in the literature. Voucher specimens have been deposited in the Louisiana State Arthropod Museum, Baton Rouge, Louisiana.

\section{Results}

The final dataset consisted of 5172 beetle specimens belonging to 55 families and 378 taxa identified to genus or species (all of which are hereafter referred to as "species", see Appendix). Of these, 47 (13\%) were non-saproxylic ground beetles, 182 (48\%) were saproxylic, and $149(39 \%)$ were other species (Appendix). Texas traps yielded the most beetles in terms of both individuals ( $44 \%$ of the total) and species $(\sim 60 \%)$ whereas Louisiana traps yielded the fewest (see Appendix; note that North Carolina traps were only sampled in 1998). State (block) was found to be a significant source of variation in most analyses of overall beetle richness, abundance and diversity in 1998 and 1999 but less so in 2002 (Table 1). The same was true when saproxylic species, ground beetles (which are non-saproxylic), and other species were analyzed separately (Table 1)

With respect to treatment differences, 2998 (58\%) individuals and $302(80 \%)$ species were collected from the dead wood plots compared to 2174 (42\%) individuals and $261(69 \%)$ species from the reference plots. Furthermore, 115 and 74 species were collected from the dead wood and reference plots, respectively. Overall, beetles were consistently more species rich, abundant, and diverse in the dead wood plots than in the reference plots during the entire study (Figure 1). Although these differences were greatest in 1998, significant differences were detected only in 1999 and only for diversity (Table 1). Similarly, only in 1999 were significant differences detected between treatments for saproxylic beetles, with them being more rich and diverse in dead wood plots than in references plots. No significant differences were detected in 2002 and non-saproxylic ground beetles and other beetle species, when analyzed separately, did not vary between treatments in any year (Table 1).

The large differences in mean beetle richness, abundance and diversity between treatments in 1998 (Figure 1) were not statistically significant due to strong interactions between state and treatment (Table 1). Most notably, the results from North Carolina were not consistent with those from the other states (Figures 2-4). After excluding North Carolina from the dataset, the differences in overall beetle richness $\left(\mathrm{F}_{1,2}=19.4, p<0.05\right)$, saproxylic beetle richness $\left(\mathrm{F}_{1,2}=23.8, p<0.05\right)$ and saproxylic beetle diversity $\left(\mathrm{F}_{1,2}=20.1, p<0.05\right)$ observed in 1998 were found to be significant. 
Table 1. Results from analyses of variance for all species combined, saproxylic species, non-saproxylic ground beetles and other species. Note that data from North Carolina are limited to 1998. Asterisks denote significant $p$-values: $*<0.05, * *<0.01, * * *<0.001$ based on analyses of variance.

\begin{tabular}{|c|c|c|c|c|c|c|c|c|c|}
\hline \multirow{2}{*}{ Parameter } & \multicolumn{3}{|c|}{$1998(n=12)$} & \multicolumn{3}{|c|}{$1999(n=9)$} & \multicolumn{3}{|c|}{$2002(n=9)$} \\
\hline & State & Treatment & State*treatment & State & Treatment & State*treatment & State & Treatment & State*treatment \\
\hline \multicolumn{10}{|l|}{ Richness } \\
\hline All species & $\mathrm{F}_{3,16}=7.5^{* *}$ & $\mathrm{~F}_{1,3}=3.4$ & $\mathrm{~F}_{3,16}=3.3^{*}$ & $\mathrm{~F}_{2,12}=16.4 * * *$ & $\mathrm{~F}_{1,2}=5.9$ & $\mathrm{~F}_{2,12}=0.1$ & $\mathrm{~F}_{2,12}=8.1 * *$ & $\mathrm{~F}_{1,2}=1.1$ & $\mathrm{~F}_{2,12}=2.3$ \\
\hline Saproxylic species & $\mathrm{F}_{3,16}=0.9$ & $\mathrm{~F}_{1,3}=6.7$ & $\mathrm{~F}_{3,16}=4.5^{*}$ & $\mathrm{~F}_{2,12}=18.0 * * *$ & $\mathrm{~F}_{1,2}=45.1^{*}$ & $\mathrm{~F}_{2,12}=0.1$ & $\mathrm{~F}_{2,12}=8.9 * *$ & $\mathrm{~F}_{1,2}=1.2$ & $\mathrm{~F}_{2,12}=1.3$ \\
\hline Ground beetles & $\mathrm{F}_{3,16}=14.3 * * *$ & $\mathrm{~F}_{1,3}=0.4$ & $\mathrm{~F}_{3,16}=0.5$ & $\mathrm{~F}_{2,12}=8.7^{* *}$ & $\mathrm{~F}_{1,2}=0.1$ & $\mathrm{~F}_{2,12}=0.5$ & $\mathrm{~F}_{2,12}=1.5$ & $\mathrm{~F}_{1,2}=1.6$ & $\mathrm{~F}_{2,12}=1.8$ \\
\hline Other species & $\mathrm{F}_{3,16}=28.6^{* * *}$ & $\mathrm{~F}_{1,3}=1.9$ & $\mathrm{~F}_{3,16}=1.1$ & $\mathrm{~F}_{2,12}=10.2 * *$ & $\mathrm{~F}_{1,2}=5.8$ & $\mathrm{~F}_{2,12}=0.1$ & $\mathrm{~F}_{2,12}=3.9$ & $\mathrm{~F}_{1,2}=0.6$ & $\mathrm{~F}_{2,12}=1.7$ \\
\hline \multicolumn{10}{|l|}{ Abundance } \\
\hline All species & $\mathrm{F}_{3,16}=13.0 * * *$ & $\mathrm{~F}_{1,3}=8.1$ & $\mathrm{~F}_{3,16}=2.2$ & $\mathrm{~F}_{2,12}=18.5^{* * *}$ & $\mathrm{~F}_{1,2}=0.4$ & $\mathrm{~F}_{2,12}=0.4$ & $\mathrm{~F}_{2,12}=2.2$ & $\mathrm{~F}_{1,2}=1.1$ & $\mathrm{~F}_{2,12}=1.9$ \\
\hline Saproxylic species & $\mathrm{F}_{3,16}=4.0^{*}$ & $\mathrm{~F}_{1,3}=8.3$ & $\mathrm{~F}_{3,16}=3.6^{*}$ & $\mathrm{~F}_{2,12}=18.7 * * *$ & $\mathrm{~F}_{1,2}=1.9$ & $\mathrm{~F}_{2,12}=0.9$ & $\mathrm{~F}_{2,12}=6.3^{*}$ & $\mathrm{~F}_{1,2}=1.7$ & $\mathrm{~F}_{2,12}=1.3$ \\
\hline Ground beetles & $\mathrm{F}_{3,16}=18.5^{* * *}$ & $\mathrm{~F}_{1,3}=0.4$ & $\mathrm{~F}_{3,16}=0.7$ & $\mathrm{~F}_{2,12}=2.7$ & $\mathrm{~F}_{1,2}=0.7$ & $\mathrm{~F}_{2,12}=0.8$ & $\mathrm{~F}_{2,12}=2.0$ & $\mathrm{~F}_{1,2}=2.4$ & $\mathrm{~F}_{2,12}=0.9$ \\
\hline Other species & $\mathrm{F}_{3,16}=11.1 * * *$ & $\mathrm{~F}_{1,3}=0.0$ & $\mathrm{~F}_{3,16}=0.4$ & $\mathrm{~F}_{2,12}=13.3 * * *$ & $\mathrm{~F}_{1,2}=0.1$ & $\mathrm{~F}_{2,12}=0.2$ & $\mathrm{~F}_{2,12}=1.7$ & $\mathrm{~F}_{1,2}=0.3$ & $\mathrm{~F}_{2,12}=2.5$ \\
\hline \multicolumn{10}{|l|}{ Diversity } \\
\hline All species & $\mathrm{F}_{3,16}=5.1^{*}$ & $\mathrm{~F}_{1,3}=2.3$ & $\mathrm{~F}_{3,16}=4.7^{*}$ & $\mathrm{~F}_{2,12}=9.9 * *$ & $\mathrm{~F}_{1,2}=147.8 * *$ & $\mathrm{~F}_{2,12}=0.0$ & $\mathrm{~F}_{2,12}=0.8$ & $\mathrm{~F}_{1,2}=1.3$ & $\mathrm{~F}_{2,12}=1.0$ \\
\hline Saproxylic species & $\mathrm{F}_{3,16}=1.5$ & $\mathrm{~F}_{1,3}=4.2$ & $\mathrm{~F}_{3,16}=4.1^{*}$ & $\mathrm{~F}_{2,12}=1.8$ & $\mathrm{~F}_{1,2}=62.4^{*}$ & $\mathrm{~F}_{2,12}=0.1$ & $\mathrm{~F}_{2,12}=1.8$ & $\mathrm{~F}_{1,2}=0.8$ & $\mathrm{~F}_{2,12}=0.6$ \\
\hline Ground beetles & $\mathrm{F}_{3,16}=7.5 * *$ & $\mathrm{~F}_{1,3}=0.1$ & $\mathrm{~F}_{3,16}=0.4$ & $\mathrm{~F}_{2,12}=16.3 * * *$ & $F_{1,2}=3.0$ & $\mathrm{~F}_{2,12}=0.2$ & $\mathrm{~F}_{2,12}=1.0$ & $\mathrm{~F}_{1,2}=1.3$ & $\mathrm{~F}_{2,12}=2.0$ \\
\hline Other species & $\mathrm{F}_{3,16}=10.2 * * *$ & $\mathrm{~F}_{1,3}=0.2$ & $\mathrm{~F}_{3,16}=1.4$ & $\mathrm{~F}_{2,12}=10.3^{* *}$ & $\mathrm{~F}_{1,2}=9.9$ & $\mathrm{~F}_{2,12}=0.2$ & $\mathrm{~F}_{2,12}=0.9$ & $\mathrm{~F}_{1,2}=0.9$ & $\mathrm{~F}_{2,12}=0.9$ \\
\hline
\end{tabular}


Figure 1. Mean $\pm \operatorname{SE}(n=12$ for 1998 and 9 for 1999 and 2002) species richness, abundance, and Shannon's diversity of beetles collected in plots in which dead wood was added or not (open and closed circles, respectively). Results for all beetle species combined are given in the left-most column followed by saproxylic species only, ground beetles only, and other species. Asterisks denote significant $p$-values: $*<0.05, * *<0.01, * * *<0.001$ based on analyses of variance.

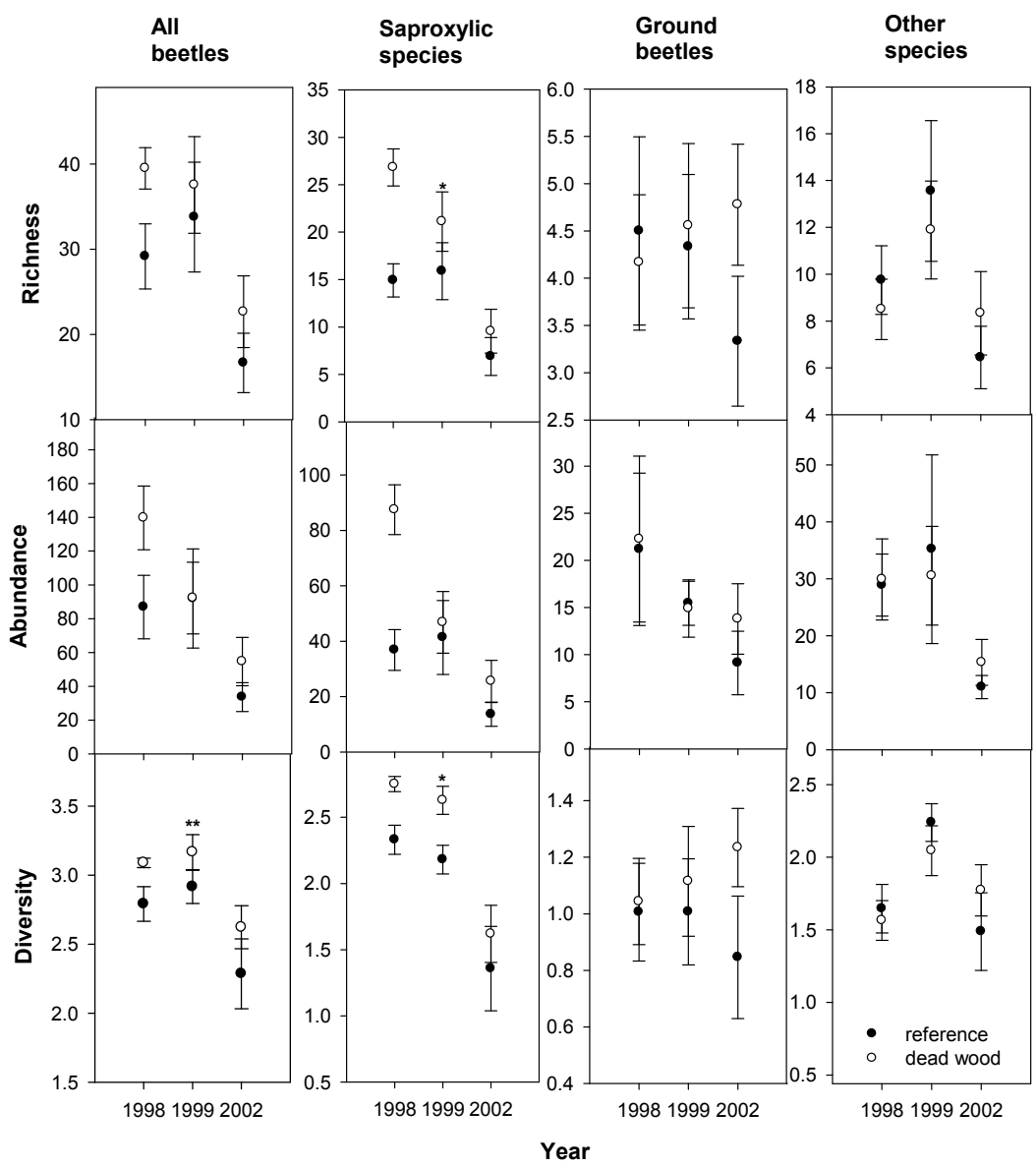

Figure 2. Mean \pm SE ( $n=3$ ) saproxylic (top) and total (bottom) beetle species richness in reference and dead wood plots (closed and open circles, respectively) by state in 1998 .

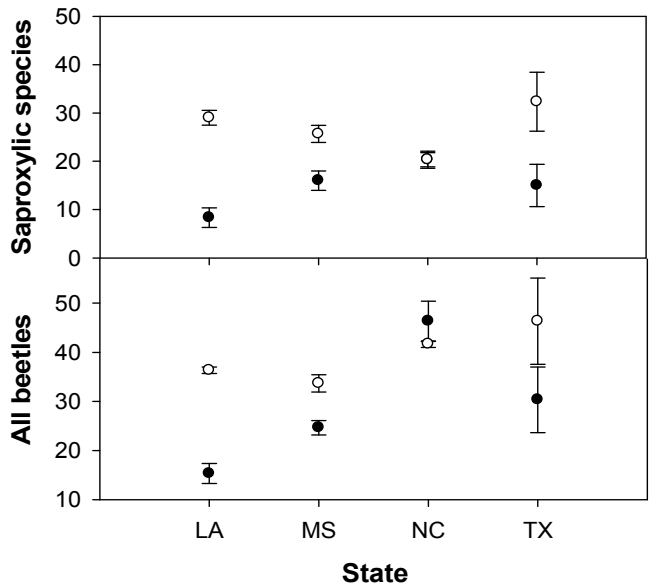


Figure 3. Mean \pm SE $(n=3)$ saproxylic (top) and total (bottom) beetle diversity (Shannon's diversity) in reference and dead wood plots (closed and open circles, respectively) by state in 1998.

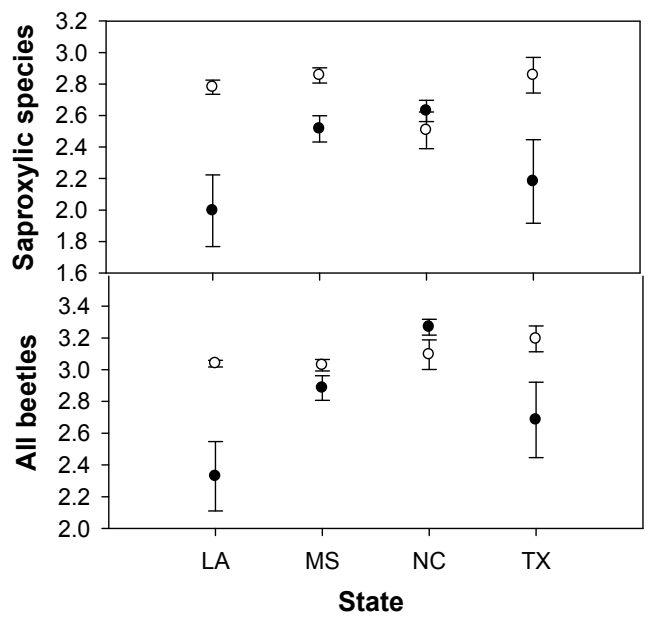

Figure 4. Mean $\pm \operatorname{SE}(n=3)$ saproxylic beetle abundance in reference and dead wood plots (closed and open circles, respectively) by state in 1998.

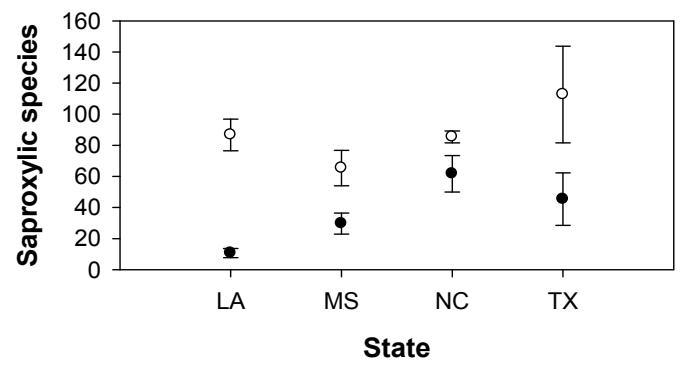

\section{Discussion}

Despite conflicting results from North Carolina which we are unable to explain based on this study, beetles generally responded positively to the dead wood manipulations made in this study, particularly during the first year. Saproxylic species were more abundant, whereas non-saproxylic ground beetles and other species did not noticeably differ. The increased abundance of saproxylic species is not surprising as these organisms, by definition, are dependent on dying and dead wood [4]. Many were no doubt captured as they attempted to colonize the fresh wood added to the plots. It is noteworthy that the degree to which saproxylic species were more numerous in the dead wood plots declined after the first year. This is largely due to the fact that more species are associated with the phloem of freshly-killed loblolly pine wood than later decay stages [29]. Once the phloem is gone, which happens quickly, so too are these early colonists. Ulyshen and Hanula [29] provided a list of beetle species found to be specifically associated with freshly-killed loblolly pine in South Carolina. Of those, the following genera were collected only during 1998 (the year the wood was added to the plots) in the current study: the cerambycids Acanthocinus and Monochamus; the zopherids Colydium and Lasconotus; the curculionids Dendroctonus, Gnathotrichus, Ips, Orthotomicus, and Myoplatypus; the 
tenebrionid Corticeus, the staphylinid Myrmecocephalus, and the histerid Platysoma (results not shown). A wide variety of other species colonize loblolly pine after the phloem stage but not in such great numbers [29]. Furthermore, many of these species are likely to re-infest the same piece of wood until it no longer provides a suitable substrate [30], thereby reducing the likelihood of being captured by pitfall traps. While the ethanol used in the trap collection jars may have attracted insects, these effects would seem to have been equal between controls and treatments, and the added wood apparently overcame any confounding effects of using this preservative.

That ground beetles were not more strongly affected by the dead wood additions is somewhat surprising considering that many previous studies have shown this group to be positively associated with dead wood [26,27,31-35]. Our results, therefore, provide little support for the idea that epigeic predators are more likely than many non-saproxylic taxa to benefit from dead wood due to its positive effect on prey abundance [27]. It is possible however that the debris in the dead wood plots impeded carabid movement enough to reduce their capture rate, thereby masking a stronger beneficial effect. Furthermore, it appears that ground beetles were becoming increasingly numerous in the dead wood plots relative to the reference plots over the course of this study (see Figure 1). Although the differences were not statistically significant, ground beetle richness and diversity had non-overlapping standard errors in 2002. These findings suggest that the benefits of dead wood to these taxa may be somewhat delayed. The results from other studies are not consistent with this conclusion, however. Within the first year after sites were clearcut in Sweden, for example, Nittérus and Gunnarsson [34] collected significantly more ground beetles in pitfall traps placed under piles of slash compared to those placed out in the open. Perhaps dead wood represents a more important source of shelter from sunlight and other desiccating conditions in clearcuts than in forested plots such as those used in the current study.

\section{Conclusions}

While some degree of caution is advised in interpreting the statistical tests we report here (due to the relatively close proximity of the sites to one another), our results are largely consistent with those of Hanula et al. [26] and Ulyshen and Hanula [27]. These studies suggest that ground-dwelling arthropods (saproxylic species notwithstanding) are little-affected by manipulations of dead wood in loblolly pine forests. This conclusion, however, is in conflict with evidence that many ground-dwelling arthropod taxa are more numerous immediately next to loblolly pine logs than short distances away from them [15]. As suggested by Ulyshen and Hanula [27], this discrepancy may indicate that dead wood has a stronger effect on how ground-dwelling arthropods are spatially distributed (i.e., causing them to become more clumped) than on their abundance or species richness. Studies aimed at addressing this question would be of particular interest. More research is also needed to determine how manipulations of dead wood at larger scales and over longer time periods affect these organisms.

\section{Acknowledgments}

We thank Jacob Carpenter, Erich Vallery, Rick Stagg, and cooperators in TX, NC, and MS for technical assistance. Victoria Bayless, Stephanie Gil, Jong-Seok Park, Igor Sokolov, and Alexey 
Tishechkin also assisted with identifications and curatorial support. We also thank Jim Hanula, Tim Schowalter and Cavell Brownie for commenting on an early version of the manuscript.

\section{Conflict of Interest}

The authors declare no conflict of interest.

\section{Appendix}

Table A1. Total beetle abundance by species, state, and treatment (reference/wood addition). Group designations are as follows: $\mathrm{S}=$ saproxylic (i.e., directly or indirectly dependent on dead wood at some life stage); $\mathrm{P}=$ predator (i.e., non-saproxylic ground beetles); $\mathrm{O}=$ other. Note that data from North Carolina are limited to 1998.

\begin{tabular}{|c|c|c|c|c|c|c|}
\hline Species & Group & Louisiana & Mississippi & North Carolina & Texas & Total \\
\hline \multicolumn{7}{|l|}{ Aderidae } \\
\hline Zonantes fasciatus (Melsheimer) & $\mathrm{S}$ & $0 / 0$ & $0 / 0$ & $1 / 0$ & $0 / 0$ & $1 / 0$ \\
\hline Zonantes signatus (Haldeman) & S & $0 / 0$ & $0 / 1$ & $0 / 0$ & $0 / 0$ & $0 / 1$ \\
\hline Zonantes subfasciatus (LeConte) & S & $0 / 0$ & $1 / 0$ & $0 / 0$ & $0 / 0$ & $1 / 0$ \\
\hline Zonantes sp. & S & $0 / 0$ & $0 / 1$ & $0 / 0$ & $0 / 0$ & $0 / 1$ \\
\hline \multicolumn{7}{|l|}{ Agyrtidae } \\
\hline Necrophilus pettitii Horn & $\mathrm{O}$ & $0 / 0$ & $5 / 2$ & $0 / 0$ & $0 / 0$ & $5 / 2$ \\
\hline \multicolumn{7}{|l|}{ Anobiidae } \\
\hline Cryptoramorphus sp. & S & $0 / 0$ & $0 / 0$ & $0 / 0$ & $0 / 1$ & $0 / 1$ \\
\hline Euvrilletta peltata (Harris) & $\mathrm{S}$ & $0 / 0$ & $0 / 0$ & $0 / 0$ & $0 / 1$ & $0 / 1$ \\
\hline Ptinus sp. & $\mathrm{S}$ & $0 / 0$ & $1 / 0$ & $0 / 0$ & $1 / 2$ & $2 / 2$ \\
\hline \multicolumn{7}{|l|}{ Anthicidae } \\
\hline Tomoderus sp. & $\mathrm{O}$ & $0 / 0$ & $2 / 0$ & $0 / 0$ & $0 / 0$ & $2 / 0$ \\
\hline Vacusus sp. & $\mathrm{O}$ & $0 / 0$ & $1 / 0$ & $0 / 0$ & $0 / 0$ & $1 / 0$ \\
\hline \multicolumn{7}{|l|}{ Attelabidae } \\
\hline Pterocolus ovatus (Fabricius) & $\mathrm{O}$ & $0 / 0$ & $0 / 0$ & $0 / 0$ & $0 / 1$ & $0 / 1$ \\
\hline \multicolumn{7}{|l|}{ Biphyllidae } \\
\hline Diplocoelus rudis (LeConte) & $\mathrm{S}$ & $8 / 7$ & $12 / 27$ & $8 / 7$ & $10 / 8$ & $38 / 49$ \\
\hline \multicolumn{7}{|l|}{ Bostrichidae } \\
\hline Lichenophanes bicornis (Weber) & S & $0 / 0$ & $0 / 0$ & $0 / 0$ & $0 / 1$ & $0 / 1$ \\
\hline Melalgus plicatus (LeConte) & S & $0 / 0$ & $0 / 0$ & $0 / 0$ & $0 / 1$ & $0 / 1$ \\
\hline Stephanopachys sp. & $\mathrm{S}$ & $0 / 0$ & $0 / 0$ & $0 / 0$ & $0 / 1$ & $0 / 1$ \\
\hline Xylobiops basilaris (Say) & S & $0 / 3$ & $1 / 2$ & $0 / 0$ & $0 / 3$ & $1 / 8$ \\
\hline \multicolumn{7}{|l|}{ Bothrideridae } \\
\hline Bothrideres cryptus Stephan & $\mathrm{S}$ & $0 / 0$ & $0 / 0$ & $0 / 0$ & $0 / 1$ & $0 / 1$ \\
\hline \multicolumn{7}{|l|}{ Brentidae } \\
\hline Sayapion segnipes (Say) & $\mathrm{O}$ & $0 / 0$ & $1 / 0$ & $0 / 0$ & $0 / 0$ & $1 / 0$ \\
\hline \multicolumn{7}{|l|}{ Buprestidae } \\
\hline Buprestis lineata Fabricius & $\mathrm{S}$ & $0 / 1$ & $0 / 0$ & $0 / 0$ & $0 / 0$ & $0 / 1$ \\
\hline Chalcophora virginiensis (Drury) & $\mathrm{S}$ & $0 / 1$ & $0 / 1$ & $0 / 0$ & $0 / 1$ & $0 / 3$ \\
\hline \multicolumn{7}{|l|}{ Cantharidae } \\
\hline Rhagonycha sp. & $\mathrm{O}$ & $3 / 0$ & $0 / 1$ & $0 / 0$ & $0 / 1$ & $3 / 2$ \\
\hline
\end{tabular}


Table A1. Cont.

\begin{tabular}{|c|c|c|c|c|c|c|}
\hline Species & Group & Louisiana & Mississippi & North Carolina & Texas & Total \\
\hline \multicolumn{7}{|l|}{ Carabidae } \\
\hline Acupalpus rectangulus Chaudoir & $\mathrm{P}$ & $0 / 0$ & $0 / 0$ & $0 / 0$ & $1 / 0$ & $1 / 0$ \\
\hline Agonum punctiforme Say & $\mathrm{P}$ & $0 / 0$ & $0 / 0$ & $0 / 0$ & $6 / 15$ & $6 / 15$ \\
\hline Amara impuncticollis (Say) & $\mathrm{P}$ & $0 / 0$ & $0 / 0$ & $0 / 0$ & $0 / 1$ & $0 / 1$ \\
\hline Anisodactylus haplomus Chaudoir & $\mathrm{P}$ & $3 / 0$ & $2 / 0$ & $0 / 0$ & $1 / 0$ & $6 / 0$ \\
\hline Apenes lucidulus Chaudoir & $\mathrm{P}$ & $0 / 0$ & $0 / 1$ & $0 / 0$ & $0 / 0$ & $0 / 1$ \\
\hline Apenes sinuatus (Say) & $\mathrm{P}$ & $0 / 0$ & $0 / 0$ & $0 / 1$ & $0 / 3$ & $0 / 4$ \\
\hline Brachinus alternans Dejean & $\mathrm{P}$ & $0 / 0$ & $0 / 0$ & $0 / 1$ & $0 / 0$ & $0 / 1$ \\
\hline Brachinus americanus (LeConte) & $\mathrm{P}$ & $0 / 0$ & $0 / 0$ & $0 / 0$ & $0 / 1$ & $0 / 1$ \\
\hline Calathus opaculus LeConte & $\mathrm{P}$ & $0 / 0$ & $0 / 0$ & $1 / 0$ & $34 / 43$ & $35 / 43$ \\
\hline Calosoma scrutator (Fabricius) & $\mathrm{P}$ & $0 / 0$ & $0 / 0$ & $0 / 0$ & $2 / 2$ & $2 / 2$ \\
\hline Carabus goryi Dejean & $\mathrm{P}$ & $0 / 0$ & $0 / 0$ & $1 / 0$ & $0 / 0$ & $1 / 0$ \\
\hline Chlaenius amoenus (Dejean) & $\mathrm{P}$ & $3 / 2$ & $2 / 2$ & $0 / 0$ & $0 / 0$ & $5 / 4$ \\
\hline Chlaenius emarginatus Say & $\mathrm{P}$ & $0 / 0$ & $0 / 0$ & $2 / 1$ & $0 / 0$ & $2 / 1$ \\
\hline Chlaenius erythropus Germar & $\mathrm{P}$ & $0 / 0$ & $0 / 0$ & $0 / 0$ & $1 / 0$ & $1 / 0$ \\
\hline Clivina ferrea LeConte & $\mathrm{P}$ & $0 / 0$ & $0 / 1$ & $0 / 0$ & $0 / 0$ & $0 / 1$ \\
\hline Clivina pallida Say & $\mathrm{P}$ & $0 / 0$ & $0 / 1$ & $0 / 0$ & $0 / 0$ & $0 / 1$ \\
\hline Coptodera aerata Dejean & $\mathrm{P}$ & $0 / 2$ & $1 / 2$ & $1 / 2$ & $1 / 2$ & $3 / 8$ \\
\hline Cyclotrachelus alabamae (Van Dyke) & $\mathrm{P}$ & $46 / 39$ & $0 / 2$ & $0 / 0$ & $23 / 24$ & $69 / 65$ \\
\hline Cyclotrachelus convivus (LeConte) & $\mathrm{P}$ & $0 / 0$ & $23 / 30$ & $0 / 0$ & $0 / 0$ & $23 / 30$ \\
\hline Cyclotrachelus laevipennis (LeConte) & $\mathrm{P}$ & $1 / 0$ & $1 / 1$ & $0 / 0$ & $0 / 0$ & $2 / 1$ \\
\hline Cyclotrachelus seximpressus (LeConte) & $\mathrm{P}$ & $1 / 1$ & $0 / 0$ & $0 / 0$ & $0 / 0$ & $1 / 1$ \\
\hline Cyclotrachelus sigillatus (Say) & $\mathrm{P}$ & $0 / 0$ & $0 / 0$ & $30 / 31$ & $0 / 0$ & $30 / 31$ \\
\hline Cyclotrachelus spoliatus (Newman) & $\mathrm{P}$ & $0 / 0$ & $0 / 0$ & $19 / 14$ & $0 / 0$ & $19 / 14$ \\
\hline Cyclotrachelus texensis (Freitag) & $\mathrm{P}$ & $6 / 5$ & $0 / 0$ & $0 / 0$ & $42 / 59$ & $48 / 64$ \\
\hline Dicaelus crenatus LeConte & $\mathrm{P}$ & $0 / 0$ & $0 / 0$ & $62 / 57$ & $0 / 0$ & $62 / 57$ \\
\hline Dicaelus elongatus Bonelli & $\mathrm{P}$ & $0 / 1$ & $5 / 9$ & $3 / 6$ & $7 / 5$ & $15 / 21$ \\
\hline Dicaelus furvus Dejean & $\mathrm{P}$ & $1 / 0$ & $1 / 4$ & $3 / 1$ & $0 / 0$ & $5 / 5$ \\
\hline Dicaelus purpuratus Bonelli & $\mathrm{P}$ & $0 / 0$ & $0 / 0$ & $3 / 1$ & $0 / 0$ & $3 / 1$ \\
\hline Elaphropus granarius (Dejean) & $\mathrm{P}$ & $0 / 2$ & $0 / 0$ & $0 / 0$ & $0 / 0$ & $0 / 2$ \\
\hline Galerita bicolor Drury & $\mathrm{P}$ & $0 / 0$ & $4 / 10$ & $57 / 82$ & $0 / 0$ & $61 / 92$ \\
\hline Harpalus rufipes Degeer & $\mathrm{P}$ & $0 / 0$ & $0 / 0$ & $0 / 0$ & $1 / 0$ & $1 / 0$ \\
\hline Harpalus sp. & $\mathrm{P}$ & $0 / 0$ & $0 / 0$ & $0 / 0$ & $1 / 0$ & $1 / 0$ \\
\hline Helluomorphoides nigripennis Dejean & $\mathrm{P}$ & $5 / 6$ & $38 / 27$ & $4 / 2$ & $0 / 0$ & $47 / 35$ \\
\hline $\begin{array}{l}\text { Helluomorphoides praestus bicolor } \\
\text { (Larochelle and Lariviere) }\end{array}$ & $\mathrm{P}$ & $0 / 0$ & $0 / 1$ & $0 / 0$ & $0 / 0$ & $0 / 1$ \\
\hline Lebia ornata Say & $\mathrm{P}$ & $0 / 0$ & $0 / 0$ & $0 / 1$ & $0 / 0$ & $0 / 1$ \\
\hline Megacephala virginica (Linneaus) & $\mathrm{P}$ & $0 / 1$ & $0 / 0$ & $0 / 0$ & $0 / 0$ & $0 / 1$ \\
\hline Mioptachys flavicauda (Say) & $\mathrm{S}$ & $1 / 1$ & $0 / 0$ & $0 / 0$ & $0 / 4$ & $1 / 5$ \\
\hline Notiophilus novemstriatus LeConte & $\mathrm{P}$ & $0 / 0$ & $6 / 6$ & $0 / 0$ & $2 / 2$ & $8 / 8$ \\
\hline Panagaeus fasciatus Say & $\mathrm{P}$ & $0 / 0$ & $0 / 0$ & $0 / 0$ & $1 / 0$ & $1 / 0$ \\
\hline Pasimachus sublaevis (Beauvois) & $\mathrm{P}$ & $0 / 0$ & $0 / 0$ & $2 / 1$ & $0 / 0$ & $2 / 1$ \\
\hline Pterostichus permundus (Say) & $\mathrm{P}$ & $0 / 0$ & $0 / 0$ & $0 / 0$ & $4 / 2$ & $4 / 2$ \\
\hline Rhadine larvalis LeConte & $\mathrm{P}$ & $0 / 0$ & $0 / 2$ & $0 / 0$ & $0 / 0$ & $0 / 2$ \\
\hline Scaphinotus cavicollis (LeConte) & $\mathrm{P}$ & $0 / 0$ & $0 / 0$ & $0 / 0$ & $4 / 5$ & $4 / 5$ \\
\hline
\end{tabular}


Table A1. Cont.

\begin{tabular}{|c|c|c|c|c|c|c|}
\hline Species & Group & Louisiana & Mississippi & North Carolina & Texas & Total \\
\hline Scaphinotus liebecki Van Dyke & $\mathrm{P}$ & $0 / 0$ & $0 / 0$ & $0 / 0$ & $1 / 0$ & $1 / 0$ \\
\hline Selenophorus ellipticus Dejean & $\mathrm{P}$ & $1 / 0$ & $0 / 0$ & $0 / 0$ & $0 / 0$ & $1 / 0$ \\
\hline Selenophorus opalinus (LeConte) & $\mathrm{P}$ & $1 / 1$ & $1 / 0$ & $0 / 0$ & $1 / 0$ & $3 / 1$ \\
\hline Selenophorus sp. & $\mathrm{P}$ & $0 / 0$ & $0 / 0$ & $0 / 0$ & $0 / 1$ & $0 / 1$ \\
\hline Sphaeroderus stenostomus (Weber) & $\mathrm{P}$ & $0 / 0$ & $0 / 0$ & $2 / 0$ & $0 / 0$ & $2 / 0$ \\
\hline Tachyta nana (Gyllenhal) & $\mathrm{S}$ & $0 / 1$ & $0 / 0$ & $0 / 0$ & $0 / 0$ & $0 / 1$ \\
\hline \multicolumn{7}{|l|}{ Cerambycidae } \\
\hline Acanthocinus obsoletus (Olivier) & $\mathrm{S}$ & $0 / 10$ & $0 / 1$ & $0 / 7$ & $0 / 2$ & $0 / 20$ \\
\hline Anelaphus pumilus (Newman) & $\mathrm{S}$ & $0 / 0$ & $0 / 0$ & $0 / 0$ & $0 / 1$ & $0 / 1$ \\
\hline Arhopalus rusticus nubilus (LeConte) & $\mathrm{S}$ & $0 / 1$ & $0 / 0$ & $0 / 0$ & $0 / 0$ & $0 / 1$ \\
\hline Astylopsis perplexa (Haldeman) & $\mathrm{S}$ & $0 / 1$ & $0 / 0$ & $0 / 0$ & $0 / 0$ & $0 / 1$ \\
\hline Curius dentatus Newman & $\mathrm{S}$ & $0 / 0$ & $1 / 0$ & $0 / 0$ & $0 / 1$ & $1 / 1$ \\
\hline Distenia undata (Fabricius) & $\mathrm{S}$ & $0 / 0$ & $0 / 0$ & $0 / 0$ & $1 / 0$ & $1 / 0$ \\
\hline Eburia quadrigeminata (Say) & $\mathrm{S}$ & $0 / 0$ & $0 / 0$ & $0 / 0$ & $1 / 0$ & $1 / 0$ \\
\hline Elaphidion mucronatum (Say) & $\mathrm{S}$ & $1 / 0$ & $0 / 1$ & $0 / 0$ & $2 / 5$ & $3 / 6$ \\
\hline Enaphalodes atomarius (Drury) & $\mathrm{S}$ & $1 / 0$ & $0 / 0$ & $0 / 0$ & $0 / 0$ & $1 / 0$ \\
\hline Eupogonius tomentosus (Haldeman) & $\mathrm{S}$ & $0 / 0$ & $1 / 0$ & $0 / 0$ & $2 / 0$ & $3 / 0$ \\
\hline Graphisurus fasciatus (DeGeer) & $\mathrm{S}$ & $0 / 1$ & $0 / 0$ & $1 / 5$ & $3 / 4$ & $4 / 10$ \\
\hline Knulliana cincta (Drury) & $\mathrm{S}$ & $0 / 0$ & $0 / 0$ & $0 / 0$ & $1 / 5$ & $1 / 5$ \\
\hline Leptostylus transversus (Gyllenhal) & $\mathrm{S}$ & $2 / 3$ & $6 / 4$ & $0 / 0$ & $12 / 28$ & $20 / 35$ \\
\hline Monochamus carolinensis (Olivier) & $\mathrm{S}$ & $0 / 0$ & $0 / 0$ & $0 / 1$ & $0 / 0$ & $0 / 1$ \\
\hline Neoclytus acuminatus (Fabricius) & $\mathrm{S}$ & $0 / 1$ & $2 / 1$ & $1 / 0$ & $10 / 0$ & $13 / 2$ \\
\hline Obrium maculatum (Olivier) & $\mathrm{S}$ & $0 / 1$ & $0 / 0$ & $0 / 0$ & $9 / 7$ & $9 / 8$ \\
\hline Orthosoma brunneum (Forster) & $\mathrm{S}$ & $0 / 0$ & $1 / 0$ & $0 / 0$ & $2 / 0$ & $3 / 0$ \\
\hline Prionus pocularis Dalman & $\mathrm{S}$ & $0 / 2$ & $1 / 13$ & $0 / 0$ & $2 / 3$ & $3 / 18$ \\
\hline Sternidius alpha (Say) & $\mathrm{S}$ & $0 / 0$ & $0 / 0$ & $3 / 1$ & $0 / 0$ & $3 / 1$ \\
\hline Styloleptus biustus (LeConte) & $\mathrm{S}$ & $0 / 0$ & $1 / 2$ & $0 / 1$ & $3 / 6$ & $4 / 9$ \\
\hline Typocerus lunulatus (Swederus) & $\mathrm{S}$ & $0 / 0$ & $0 / 0$ & $0 / 0$ & $1 / 0$ & $1 / 0$ \\
\hline Typocerus velutinus (Olivier) & $\mathrm{S}$ & $0 / 0$ & $1 / 0$ & $0 / 0$ & $0 / 0$ & $1 / 0$ \\
\hline Typocerus zebra (Olivier) & $\mathrm{S}$ & $0 / 0$ & $2 / 2$ & $1 / 0$ & $0 / 0$ & $3 / 2$ \\
\hline Xylotrechus colonus (Fabricius) & $\mathrm{S}$ & $0 / 0$ & $0 / 0$ & $0 / 0$ & $3 / 4$ & $3 / 4$ \\
\hline Xylotrechus s. sagittatus (Germar) & $\mathrm{S}$ & $0 / 0$ & $0 / 1$ & $0 / 0$ & $0 / 0$ & $0 / 1$ \\
\hline \multicolumn{7}{|l|}{ Cerylonidae } \\
\hline Philothermus glabriculus LeConte & $\mathrm{S}$ & $0 / 0$ & $0 / 1$ & $0 / 0$ & $0 / 1$ & $0 / 2$ \\
\hline \multicolumn{7}{|l|}{ Chrysomelidae } \\
\hline Capraita circumdata (Randall) & $\mathrm{O}$ & $0 / 0$ & $0 / 3$ & $0 / 0$ & $0 / 1$ & $0 / 4$ \\
\hline Capraita obsidiana (Fabricius) & $\mathrm{O}$ & $0 / 0$ & $0 / 0$ & $0 / 0$ & $0 / 1$ & $0 / 1$ \\
\hline Capraita suturalis (Fabricius) & $\mathrm{O}$ & $0 / 0$ & $0 / 2$ & $0 / 0$ & $0 / 0$ & $0 / 2$ \\
\hline Capraita thyamoides (Crotch) & $\mathrm{O}$ & $0 / 0$ & $0 / 0$ & $0 / 0$ & $3 / 1$ & $3 / 1$ \\
\hline Capraita sp. & $\mathrm{O}$ & $0 / 0$ & $0 / 0$ & $2 / 0$ & $0 / 3$ & $2 / 3$ \\
\hline Graphops curtipennis (Melsheimer) & $\mathrm{O}$ & $3 / 1$ & $0 / 0$ & $0 / 0$ & $0 / 0$ & $3 / 1$ \\
\hline Graphops floridanus Blake & $\mathrm{O}$ & $2 / 1$ & $0 / 0$ & $0 / 0$ & $0 / 0$ & $2 / 1$ \\
\hline Metachroma pellucidum Crotch & $\mathrm{O}$ & $0 / 0$ & $0 / 2$ & $0 / 0$ & $0 / 0$ & $0 / 2$ \\
\hline Orthaltica copalina (Fabricius) & $\mathrm{O}$ & $0 / 0$ & $1 / 0$ & $0 / 0$ & $0 / 0$ & $1 / 0$ \\
\hline Paria sp. & $\mathrm{O}$ & $0 / 4$ & $0 / 0$ & $0 / 0$ & $12 / 3$ & $12 / 7$ \\
\hline
\end{tabular}


Table A1. Cont.

\begin{tabular}{|c|c|c|c|c|c|c|}
\hline Species & Group & Louisiana & Mississippi & North Carolina & Texas & Total \\
\hline Rhabdopterus sp. & $\mathrm{O}$ & $0 / 0$ & $3 / 0$ & $2 / 1$ & $0 / 0$ & $5 / 1$ \\
\hline \multicolumn{7}{|l|}{ Ciidae } \\
\hline Cis sp. & $\mathrm{S}$ & $0 / 0$ & $0 / 0$ & $0 / 0$ & $1 / 1$ & $1 / 1$ \\
\hline \multicolumn{7}{|l|}{ Cleridae } \\
\hline Cymatodera wolcotti Barr & $\mathrm{S}$ & $1 / 0$ & $0 / 0$ & $0 / 0$ & $0 / 0$ & $1 / 0$ \\
\hline Neorthopleura thoracica (Say) & $\mathrm{S}$ & $0 / 1$ & $0 / 2$ & $0 / 0$ & $1 / 2$ & $1 / 5$ \\
\hline Priocera castanea (Newman) & $\mathrm{S}$ & $0 / 5$ & $0 / 1$ & $0 / 0$ & $0 / 0$ & $0 / 6$ \\
\hline \multicolumn{7}{|l|}{ Coccinellidae } \\
\hline Psyllobora vigintimaculata (Say) & $\mathrm{O}$ & $0 / 0$ & $0 / 0$ & $1 / 0$ & $0 / 0$ & $1 / 0$ \\
\hline \multicolumn{7}{|l|}{ Corylophidae } \\
\hline Arthrolips fasciata (Erichson) & $\mathrm{S}$ & $0 / 0$ & $0 / 0$ & $0 / 0$ & $0 / 3$ & $0 / 3$ \\
\hline Clypastracea sp. & $\mathrm{S}$ & $0 / 0$ & $0 / 0$ & $0 / 0$ & $0 / 1$ & $0 / 1$ \\
\hline \multicolumn{7}{|l|}{ Cryptophagidae } \\
\hline Cryptophagus sp. & $\mathrm{S}$ & $1 / 0$ & $0 / 0$ & $0 / 0$ & $0 / 1$ & $1 / 1$ \\
\hline \multicolumn{7}{|l|}{ Curculionidae } \\
\hline Acalles clavatus (Say) & $\mathrm{S}$ & $0 / 1$ & $3 / 19$ & $3 / 3$ & $1 / 2$ & $7 / 25$ \\
\hline Ambrosiodmus rubricolllis (Eichhoff) & $\mathrm{S}$ & $1 / 1$ & $5 / 8$ & $11 / 13$ & $3 / 5$ & $20 / 27$ \\
\hline Apteromechus ferratus (Say) & $\mathrm{S}$ & $0 / 0$ & $0 / 4$ & $1 / 1$ & $13 / 27$ & $14 / 32$ \\
\hline Coccotrypes distinctus (Motschulsky) & $\mathrm{S}$ & $0 / 1$ & $0 / 0$ & $0 / 0$ & $0 / 0$ & $0 / 1$ \\
\hline Conotrachelus posticatus Boheman & $\mathrm{O}$ & $0 / 0$ & $0 / 0$ & $1 / 0$ & $103 / 68$ & $104 / 68$ \\
\hline Cophes fallax (LeConte) & $\mathrm{S}$ & $0 / 0$ & $0 / 0$ & $0 / 0$ & $1 / 3$ & $1 / 3$ \\
\hline Corthylus punctatissimus (Zimmermann) & $\mathrm{S}$ & $0 / 0$ & $1 / 0$ & $0 / 1$ & $0 / 0$ & $1 / 1$ \\
\hline Cossonus corticola Say & $\mathrm{S}$ & $0 / 13$ & $1 / 6$ & $0 / 0$ & $5 / 41$ & $6 / 60$ \\
\hline Cryptorhynchus tristis LeConte & $\mathrm{O}$ & $1 / 2$ & $2 / 3$ & $0 / 0$ & $1 / 0$ & $4 / 5$ \\
\hline Cyrtepistomus castaneus (Roelofs) & $\mathrm{O}$ & $0 / 0$ & $1 / 0$ & $0 / 0$ & $0 / 0$ & $1 / 0$ \\
\hline Dendroctonus terebrans (Olivier) & $\mathrm{S}$ & $0 / 0$ & $0 / 0$ & $0 / 0$ & $0 / 6$ & $0 / 6$ \\
\hline Dryophthorus americanus Bedel & $\mathrm{S}$ & $0 / 2$ & $2 / 3$ & $10 / 0$ & $4 / 3$ & $16 / 8$ \\
\hline Dryoxylon onoharaensis (Murayama) & $\mathrm{S}$ & $0 / 0$ & $1 / 4$ & $0 / 0$ & $0 / 0$ & $1 / 4$ \\
\hline Euplatypus compositus (Say) & $\mathrm{S}$ & $4 / 7$ & $0 / 2$ & $0 / 0$ & $1 / 1$ & $5 / 10$ \\
\hline Euwallacea validus (Eichhoff) & $\mathrm{S}$ & $0 / 0$ & $0 / 1$ & $0 / 0$ & $0 / 0$ & $0 / 1$ \\
\hline Gnathotrichus materiarius (Fitch) & $\mathrm{S}$ & $0 / 0$ & $0 / 0$ & $0 / 3$ & $0 / 1$ & $0 / 4$ \\
\hline Hylastes porculus Erichson & $\mathrm{S}$ & $0 / 2$ & $0 / 0$ & $0 / 1$ & $0 / 1$ & $0 / 4$ \\
\hline Hylastes salebrosus Eichhoff & $\mathrm{S}$ & $1 / 2$ & $0 / 0$ & $0 / 0$ & $0 / 2$ & $1 / 4$ \\
\hline Hylastes tenuis Eichhoff & $\mathrm{S}$ & $1 / 2$ & $1 / 9$ & $6 / 9$ & $1 / 9$ & $9 / 29$ \\
\hline Hylobius pales (Herbst) & $\mathrm{S}$ & $2 / 8$ & $8 / 12$ & $11 / 30$ & $3 / 12$ & $24 / 62$ \\
\hline Hypothenemus sp. & $\mathrm{S}$ & $0 / 0$ & $0 / 0$ & $1 / 1$ & $0 / 1$ & $1 / 2$ \\
\hline Ips avulsus (Eichhoff) & $\mathrm{S}$ & $0 / 2$ & $1 / 1$ & $0 / 0$ & $1 / 5$ & $2 / 8$ \\
\hline Ips calligraphus (Germar) & $\mathrm{S}$ & $0 / 1$ & $0 / 1$ & $0 / 0$ & $0 / 2$ & $0 / 4$ \\
\hline Ips grandicollis (Eichhoff) & $\mathrm{S}$ & $0 / 0$ & $0 / 0$ & $0 / 0$ & $0 / 4$ & $0 / 4$ \\
\hline Lissorhoptrus oryzophilus Kuschel & $\mathrm{O}$ & $0 / 1$ & $0 / 0$ & $0 / 0$ & $0 / 0$ & $0 / 1$ \\
\hline Lissorhoptrus simplex (Say) & $\mathrm{O}$ & $1 / 0$ & $0 / 0$ & $0 / 0$ & $0 / 0$ & $1 / 0$ \\
\hline Monarthrum fasciatum (Say) & $\mathrm{S}$ & $0 / 0$ & $0 / 0$ & $0 / 0$ & $0 / 1$ & $0 / 1$ \\
\hline Monarthrum mali (Fitch) & $\mathrm{S}$ & $0 / 0$ & $0 / 0$ & $0 / 0$ & $2 / 4$ & $2 / 4$ \\
\hline Myoplatypus flavicornis (Fabricius) & $\mathrm{S}$ & $0 / 15$ & $0 / 3$ & $0 / 25$ & $0 / 19$ & $0 / 62$ \\
\hline Notaris puncticollis (LeConte) & $\mathrm{O}$ & $0 / 0$ & $1 / 0$ & $0 / 0$ & $0 / 0$ & $1 / 0$ \\
\hline
\end{tabular}


Table A1. Cont.

\begin{tabular}{|c|c|c|c|c|c|c|}
\hline Species & Group & Louisiana & Mississippi & North Carolina & Texas & Total \\
\hline Orthotomicus caelatus (Eichhoff) & $\mathrm{S}$ & $0 / 38$ & $7 / 10$ & $11 / 52$ & $25 / 59$ & $43 / 159$ \\
\hline Pachylobius picivorus (Germar) & $\mathrm{S}$ & $5 / 30$ & $10 / 38$ & $4 / 13$ & $25 / 40$ & $44 / 121$ \\
\hline Pityophthorus confusus Blandford & $\mathrm{S}$ & $0 / 1$ & $0 / 0$ & $0 / 0$ & $0 / 0$ & $0 / 1$ \\
\hline Sphenophorus sp. & $\mathrm{O}$ & $0 / 0$ & $0 / 0$ & $0 / 0$ & $1 / 0$ & $1 / 0$ \\
\hline Stethobaris sp. & $\mathrm{O}$ & $1 / 1$ & $0 / 0$ & $0 / 0$ & $0 / 0$ & $1 / 1$ \\
\hline Xyleborinus saxeseni (Ratzeburg) & $\mathrm{S}$ & $14 / 10$ & $22 / 12$ & $9 / 2$ & $117 / 98$ & $162 / 122$ \\
\hline Xyleborus affinis Eichhoff & $\mathrm{S}$ & $4 / 36$ & $28 / 51$ & $27 / 18$ & $13 / 60$ & $72 / 165$ \\
\hline Xyleborus ferrugineus (Fabricius) & $\mathrm{S}$ & $3 / 33$ & $6 / 16$ & $23 / 10$ & $39 / 58$ & $71 / 117$ \\
\hline Xyleborus pubescens Zimmermann & $\mathrm{S}$ & $1 / 3$ & $0 / 0$ & $1 / 1$ & $1 / 5$ & $3 / 9$ \\
\hline Xyleborus xylographus (Say) & $\mathrm{S}$ & $0 / 0$ & $0 / 0$ & $1 / 0$ & $0 / 1$ & $1 / 1$ \\
\hline Xylosandrus compactus (Eichhoff) & $\mathrm{S}$ & $0 / 0$ & $1 / 1$ & $22 / 30$ & $1 / 2$ & $24 / 33$ \\
\hline Xylosandrus crassiusculus (Motschulsky) & $\mathrm{S}$ & $1 / 4$ & $9 / 17$ & $9 / 9$ & $0 / 5$ & $19 / 35$ \\
\hline Xylosandrus germanus (Blandford) & $\mathrm{S}$ & $1 / 0$ & $4 / 7$ & $0 / 0$ & $0 / 0$ & $5 / 7$ \\
\hline \multicolumn{7}{|l|}{ Dytiscidae } \\
\hline Copelatus glyphicus (Say) & $\mathrm{O}$ & $5 / 1$ & $0 / 0$ & $1 / 1$ & $1 / 1$ & $7 / 3$ \\
\hline \multicolumn{7}{|l|}{ Elateridae } \\
\hline Alaus myops (Fabricius) & $\mathrm{S}$ & $0 / 1$ & $0 / 1$ & $0 / 0$ & $0 / 0$ & $0 / 2$ \\
\hline Blauta cribraria (Germar) & $\mathrm{S}$ & $0 / 3$ & $0 / 1$ & $0 / 0$ & $0 / 0$ & $0 / 4$ \\
\hline Conoderus amplicollis (Gyllenhal) & $\mathrm{S}$ & $0 / 1$ & $0 / 0$ & $0 / 0$ & $0 / 0$ & $0 / 1$ \\
\hline Dicrepidius sp. & $\mathrm{S}$ & $0 / 0$ & $0 / 0$ & $0 / 0$ & $0 / 1$ & $0 / 1$ \\
\hline Glyphonyx bimarginatus Schaeffer & $\mathrm{S}$ & $0 / 0$ & $0 / 0$ & $0 / 0$ & $1 / 0$ & $1 / 0$ \\
\hline Glyphonyx ferruginosus Schaeffer & $\mathrm{S}$ & $0 / 0$ & $0 / 0$ & $0 / 0$ & $1 / 1$ & $1 / 1$ \\
\hline Glyphonyx sp. & $\mathrm{S}$ & $0 / 0$ & $0 / 0$ & $0 / 1$ & $0 / 0$ & $0 / 1$ \\
\hline Lacon discoideus (Weber) & $\mathrm{S}$ & $0 / 0$ & $0 / 1$ & $0 / 0$ & $0 / 0$ & $0 / 1$ \\
\hline Lacon impressicollis (Say) & $\mathrm{S}$ & $0 / 0$ & $0 / 0$ & $0 / 0$ & $0 / 1$ & $0 / 1$ \\
\hline Limonius quercinus Say & $\mathrm{S}$ & $0 / 0$ & $0 / 0$ & $1 / 0$ & $0 / 0$ & $1 / 0$ \\
\hline Megapenthes rufilabris (Germar) & $\mathrm{S}$ & $1 / 0$ & $0 / 1$ & $0 / 0$ & $0 / 1$ & $1 / 2$ \\
\hline Megapenthes sp. & $\mathrm{S}$ & $0 / 0$ & $0 / 0$ & $0 / 1$ & $0 / 0$ & $0 / 1$ \\
\hline Melanotus corticinus (Say) & $\mathrm{S}$ & $0 / 0$ & $0 / 1$ & $0 / 0$ & $0 / 0$ & $0 / 1$ \\
\hline Melanotus ignobilis Melsheimer & $\mathrm{S}$ & $1 / 2$ & $0 / 0$ & $0 / 0$ & $0 / 0$ & $1 / 2$ \\
\hline Melanotus insipiens (Say) & $\mathrm{S}$ & $0 / 0$ & $0 / 0$ & $0 / 0$ & $1 / 0$ & $1 / 0$ \\
\hline Melanotus piceatus Blatchley & $\mathrm{S}$ & $0 / 0$ & $0 / 0$ & $2 / 0$ & $0 / 0$ & $2 / 0$ \\
\hline Melanotus pilosus Blatchley & $\mathrm{S}$ & $1 / 1$ & $0 / 0$ & $0 / 0$ & $0 / 0$ & $1 / 1$ \\
\hline Melanotus similis group & $\mathrm{S}$ & $0 / 0$ & $1 / 1$ & $0 / 1$ & $0 / 0$ & $1 / 2$ \\
\hline Melanotus testaceus (Melsheimer) & $\mathrm{S}$ & $0 / 0$ & $0 / 0$ & $0 / 0$ & $1 / 0$ & $1 / 0$ \\
\hline Melanotus sp. & $\mathrm{S}$ & $0 / 0$ & $2 / 2$ & $0 / 1$ & $0 / 1$ & $2 / 4$ \\
\hline Mulsanteus carolinensis (Schaeffer) & $\mathrm{S}$ & $0 / 0$ & $0 / 0$ & $0 / 0$ & $13 / 2$ & $13 / 2$ \\
\hline \multicolumn{7}{|l|}{ Endomychidae } \\
\hline Aphorista vittata (Fabricius) & $\mathrm{S}$ & $1 / 2$ & $2 / 3$ & $0 / 0$ & $4 / 7$ & $7 / 12$ \\
\hline Danae testacea (Ziegler) & $\mathrm{S}$ & $0 / 1$ & $2 / 3$ & $7 / 3$ & $4 / 3$ & $13 / 10$ \\
\hline Epipocus punctatus LeConte & $\mathrm{S}$ & $1 / 1$ & $0 / 0$ & $0 / 0$ & $0 / 0$ & $1 / 1$ \\
\hline Lycoperdina ferruginea LeConte & $\mathrm{S}$ & $0 / 0$ & $4 / 2$ & $1 / 0$ & $8 / 12$ & $13 / 14$ \\
\hline Mycetina perpulchra (Newman) & $\mathrm{S}$ & $0 / 0$ & $0 / 1$ & $0 / 0$ & $0 / 0$ & $0 / 1$ \\
\hline
\end{tabular}


Table A1. Cont.

\begin{tabular}{|c|c|c|c|c|c|c|}
\hline Species & Group & Louisiana & Mississippi & North Carolina & Texas & Total \\
\hline \multicolumn{7}{|l|}{ Erotylidae } \\
\hline Cryptophilus integer (Heer) & $\mathrm{S}$ & $0 / 0$ & $0 / 1$ & $0 / 0$ & $0 / 0$ & $0 / 1$ \\
\hline Triplax festiva Lacordaire & $\mathrm{S}$ & $0 / 0$ & $0 / 0$ & $0 / 0$ & $1 / 0$ & $1 / 0$ \\
\hline Tritoma affinis Lacordaire & $\mathrm{S}$ & $2 / 3$ & $11 / 3$ & $0 / 0$ & $8 / 11$ & $21 / 17$ \\
\hline Tritoma angulata Say & $\mathrm{S}$ & $0 / 0$ & $1 / 0$ & $0 / 0$ & $0 / 0$ & $1 / 0$ \\
\hline Tritoma atriventris LeConte & $\mathrm{S}$ & $0 / 0$ & $0 / 0$ & $0 / 0$ & $1 / 3$ & $1 / 3$ \\
\hline Tritoma biguttata affinis Lacordaire & $\mathrm{S}$ & $1 / 2$ & $4 / 4$ & $0 / 0$ & $4 / 3$ & $9 / 9$ \\
\hline Tritoma humeralis Fabricius & $\mathrm{S}$ & $0 / 0$ & $0 / 0$ & $0 / 3$ & $0 / 0$ & $0 / 3$ \\
\hline \multicolumn{7}{|l|}{ Eucinetidae } \\
\hline Eucinetus strigosus LeConte & $\mathrm{S}$ & $1 / 4$ & $4 / 7$ & $0 / 0$ & $0 / 0$ & $5 / 11$ \\
\hline \multicolumn{7}{|l|}{ Eucnemidae } \\
\hline Dromaeolus cylindricollis (Say) & $\mathrm{S}$ & $5 / 0$ & $1 / 2$ & $0 / 0$ & $1 / 2$ & $7 / 4$ \\
\hline Dromaeolus striatus (LeConte) & $\mathrm{S}$ & $1 / 1$ & $2 / 2$ & $0 / 0$ & $0 / 0$ & $3 / 3$ \\
\hline Microrhagus triangularis (Say) & $\mathrm{S}$ & $0 / 0$ & $0 / 0$ & $0 / 0$ & $2 / 1$ & $2 / 1$ \\
\hline \multicolumn{7}{|l|}{ Geotrupidae } \\
\hline Bolboceras thoracicornis (Wallis) & $\mathrm{O}$ & $0 / 0$ & $0 / 0$ & $1 / 0$ & $0 / 0$ & $1 / 0$ \\
\hline Bolbocerosoma farctum (Fabricius) & $\mathrm{O}$ & $0 / 0$ & $1 / 1$ & $0 / 0$ & $0 / 0$ & $1 / 1$ \\
\hline Geotrupes blackburnii (Fabricius) & $\mathrm{O}$ & $1 / 0$ & $0 / 0$ & $0 / 0$ & $1 / 3$ & $2 / 3$ \\
\hline Geotrupes opacus Haldeman & $\mathrm{O}$ & $0 / 0$ & $0 / 0$ & $0 / 1$ & $6 / 0$ & $6 / 1$ \\
\hline Odonteus sp. & $\mathrm{O}$ & $0 / 0$ & $0 / 0$ & $1 / 0$ & $0 / 0$ & $1 / 0$ \\
\hline \multicolumn{7}{|l|}{ Histeridae } \\
\hline Eblisia carolina (Paykull) & $\mathrm{S}$ & $0 / 0$ & $0 / 0$ & $0 / 0$ & $0 / 1$ & $0 / 1$ \\
\hline Paromalus seminulum Erichson & $\mathrm{S}$ & $0 / 1$ & $1 / 0$ & $0 / 0$ & $0 / 1$ & $1 / 2$ \\
\hline Platysoma coarctatum LeConte & $\mathrm{S}$ & $0 / 0$ & $0 / 1$ & $0 / 0$ & $0 / 2$ & $0 / 3$ \\
\hline \multicolumn{7}{|l|}{ Hydrophilidae } \\
\hline Cercyon occallatus (Say) & $\mathrm{O}$ & $0 / 0$ & $1 / 0$ & $9 / 4$ & $0 / 0$ & $10 / 4$ \\
\hline Cercyon pubescens LeConte & $\mathrm{O}$ & $1 / 0$ & $0 / 0$ & $0 / 0$ & $3 / 0$ & $4 / 0$ \\
\hline Cymbiodyta chamberlaini Smetana & $\mathrm{O}$ & $1 / 0$ & $0 / 0$ & $0 / 0$ & $0 / 1$ & $1 / 1$ \\
\hline \multicolumn{7}{|l|}{ Laemophloeidae } \\
\hline Cryptolestes punctatus (LeConte) & $\mathrm{S}$ & $0 / 1$ & $0 / 0$ & $0 / 0$ & $0 / 0$ & $0 / 1$ \\
\hline Cryptolestes sp. & $\mathrm{S}$ & $0 / 0$ & $0 / 0$ & $0 / 0$ & $0 / 1$ & $0 / 1$ \\
\hline Laemophloeus biguttatus Say & $\mathrm{S}$ & $0 / 0$ & $0 / 1$ & $0 / 0$ & $0 / 0$ & $0 / 1$ \\
\hline Placonotus modestus (Say) & $\mathrm{S}$ & $0 / 0$ & $0 / 2$ & $0 / 0$ & $4 / 2$ & $4 / 4$ \\
\hline \multicolumn{7}{|l|}{ Lampyridae } \\
\hline Ellychnia corrusca (LeConte) & $\mathrm{S}$ & $0 / 0$ & $0 / 0$ & $1 / 0$ & $0 / 0$ & $1 / 0$ \\
\hline \multicolumn{7}{|l|}{ Latridiidae } \\
\hline Aridius sp. & $\mathrm{S}$ & $0 / 0$ & $0 / 0$ & $0 / 1$ & $0 / 0$ & $0 / 1$ \\
\hline Corticarina sp. & $\mathrm{S}$ & $1 / 2$ & $0 / 0$ & $0 / 0$ & $0 / 0$ & $1 / 2$ \\
\hline \multicolumn{7}{|l|}{ Leiodidae } \\
\hline Anisotoma basalis (LeConte) & $\mathrm{O}$ & $0 / 0$ & $0 / 0$ & $1 / 0$ & $0 / 0$ & $1 / 0$ \\
\hline Anisotoma discolor (Melsheimer) & $\mathrm{O}$ & $0 / 0$ & $0 / 0$ & $1 / 0$ & $1 / 5$ & $2 / 5$ \\
\hline Anisotoma sp. & $\mathrm{O}$ & $0 / 0$ & $0 / 0$ & $0 / 0$ & $1 / 0$ & $1 / 0$ \\
\hline Colenis bifida Peck & $\mathrm{O}$ & $0 / 0$ & $0 / 0$ & $2 / 0$ & $0 / 0$ & $2 / 0$ \\
\hline Colenis impunctata LeConte & $\mathrm{O}$ & $2 / 10$ & $0 / 1$ & $5 / 8$ & $19 / 16$ & $26 / 35$ \\
\hline Colenis ora Peck & $\mathrm{O}$ & $0 / 1$ & $2 / 1$ & $0 / 0$ & $15 / 20$ & $17 / 22$ \\
\hline
\end{tabular}


Table A1. Cont.

\begin{tabular}{|c|c|c|c|c|c|c|}
\hline Species & Group & Louisiana & Mississippi & North Carolina & Texas & Total \\
\hline Colenis stephani Peck & $\mathrm{O}$ & $0 / 0$ & $0 / 0$ & $0 / 0$ & $2 / 1$ & $2 / 1$ \\
\hline Colenis sp. & $\mathrm{O}$ & $0 / 1$ & $0 / 0$ & $0 / 1$ & $0 / 0$ & $0 / 2$ \\
\hline Dissochaetus oblitus (LeConte) & $\mathrm{O}$ & $0 / 0$ & $0 / 0$ & $0 / 1$ & $0 / 0$ & $0 / 1$ \\
\hline Leiodes stephani Baranowski & $\mathrm{O}$ & $0 / 0$ & $0 / 0$ & $0 / 0$ & $4 / 0$ & $4 / 0$ \\
\hline Ptomaphagus consobrinus LeConte & $\mathrm{O}$ & $4 / 5$ & $0 / 0$ & $0 / 0$ & $3 / 5$ & $7 / 10$ \\
\hline Ptomaphagus sp. & $\mathrm{O}$ & $0 / 0$ & $0 / 0$ & $0 / 0$ & $1 / 0$ & $1 / 0$ \\
\hline \multicolumn{7}{|l|}{ Lucanidae } \\
\hline Dorcus parallelus (Say) & $\mathrm{S}$ & $0 / 0$ & $0 / 0$ & $1 / 1$ & $0 / 0$ & $1 / 1$ \\
\hline \multicolumn{7}{|l|}{ Lycidae } \\
\hline Plateros sp. & $\mathrm{S}$ & $0 / 2$ & $1 / 3$ & $0 / 0$ & $1 / 0$ & $2 / 5$ \\
\hline \multicolumn{7}{|l|}{ Melandryidae } \\
\hline Dircaea liturata (LeConte) & $\mathrm{S}$ & $0 / 0$ & $0 / 0$ & $0 / 0$ & $0 / 1$ & $0 / 1$ \\
\hline Microtonus sericans LeConte & $\mathrm{S}$ & $0 / 0$ & $1 / 0$ & $0 / 0$ & $0 / 0$ & $1 / 0$ \\
\hline \multicolumn{7}{|l|}{ Mordellidae } \\
\hline Mordella sp. & $\mathrm{O}$ & $0 / 0$ & $1 / 1$ & $0 / 0$ & $0 / 1$ & $1 / 2$ \\
\hline Mordellaria borealis (LeConte) & $\mathrm{O}$ & $0 / 1$ & $1 / 0$ & $0 / 0$ & $0 / 0$ & $1 / 1$ \\
\hline Mordellaria serval (Say) & $\mathrm{O}$ & $0 / 0$ & $1 / 0$ & $0 / 0$ & $1 / 3$ & $2 / 3$ \\
\hline Mordellistena pubescens (Fabricius) & $\mathrm{O}$ & $0 / 0$ & $0 / 1$ & $0 / 0$ & $0 / 0$ & $0 / 1$ \\
\hline \multicolumn{7}{|l|}{ Mycetophagidae } \\
\hline Typhaea stercorea (Linnaeus) & $\mathrm{S}$ & $0 / 2$ & $1 / 0$ & $0 / 0$ & $17 / 19$ & $18 / 21$ \\
\hline \multicolumn{7}{|l|}{ Nitidulidae } \\
\hline Amphicrossus ciliatus (Olivier) & $\mathrm{O}$ & $0 / 0$ & $0 / 1$ & $0 / 0$ & $0 / 0$ & $0 / 1$ \\
\hline Carpophilus antiquus Melsheimer & $\mathrm{O}$ & $0 / 0$ & $0 / 0$ & $1 / 1$ & $1 / 0$ & $2 / 1$ \\
\hline Carpophilus sp. & $\mathrm{O}$ & $1 / 1$ & $0 / 2$ & $1 / 4$ & $3 / 2$ & $5 / 9$ \\
\hline Colopterus unicolor (Say) & $\mathrm{O}$ & $10 / 16$ & $12 / 39$ & $43 / 55$ & $30 / 66$ & $95 / 176$ \\
\hline Epuraea helvola Erichson & $\mathrm{O}$ & $0 / 0$ & $1 / 0$ & $3 / 13$ & $0 / 0$ & $4 / 13$ \\
\hline Epuraea planulata Erichson & $\mathrm{O}$ & $0 / 0$ & $0 / 0$ & $0 / 1$ & $0 / 0$ & $0 / 1$ \\
\hline Epuraea rufa (Say) & $\mathrm{O}$ & $0 / 0$ & $0 / 0$ & $7 / 4$ & $0 / 0$ & $7 / 4$ \\
\hline Glischrochilus sanguinolentus (Olivier) & $\mathrm{O}$ & $0 / 0$ & $1 / 2$ & $0 / 0$ & $0 / 0$ & $1 / 2$ \\
\hline Pallodes austrinus Leschen & $\mathrm{O}$ & $0 / 0$ & $2 / 0$ & $1 / 0$ & $1 / 2$ & $4 / 2$ \\
\hline Pallodes pallidus Beauvois & $\mathrm{O}$ & $0 / 0$ & $1 / 3$ & $1 / 3$ & $0 / 0$ & $2 / 6$ \\
\hline Prometopia sexmaculata (Say) & $\mathrm{O}$ & $0 / 1$ & $0 / 0$ & $0 / 0$ & $1 / 0$ & $1 / 1$ \\
\hline Stelidota coenosa Erichson & $\mathrm{O}$ & $8 / 7$ & $2 / 3$ & $1 / 1$ & $23 / 22$ & $34 / 33$ \\
\hline Stelidota geminata (Say) & $\mathrm{O}$ & $1 / 3$ & $0 / 1$ & $7 / 12$ & $6 / 3$ & $14 / 19$ \\
\hline Stelidota octomaculata (Say) & $\mathrm{O}$ & $0 / 0$ & $0 / 0$ & $0 / 0$ & $0 / 2$ & $0 / 2$ \\
\hline Stelidota sexmaculata (Say) & $\mathrm{O}$ & $0 / 0$ & $0 / 0$ & $0 / 0$ & $1 / 0$ & $1 / 0$ \\
\hline Stelidota strigosa Schoenherr & $\mathrm{O}$ & $0 / 0$ & $1 / 0$ & $0 / 0$ & $6 / 3$ & $7 / 3$ \\
\hline \multicolumn{7}{|l|}{ Oedemeridae } \\
\hline Oxycopis notoxoides (Fabricius) & $\mathrm{O}$ & $1 / 0$ & $0 / 0$ & $0 / 0$ & $0 / 0$ & $1 / 0$ \\
\hline Oxycopis sp. & $\mathrm{S}$ & $0 / 0$ & $0 / 0$ & $0 / 0$ & $1 / 0$ & $1 / 0$ \\
\hline Oxycopis thoracica (Fabricius) & $\mathrm{S}$ & $0 / 0$ & $0 / 1$ & $0 / 0$ & $0 / 0$ & $0 / 1$ \\
\hline \multicolumn{7}{|l|}{ Passandridae } \\
\hline Catogenus rufus (Fabricius) & $\mathrm{S}$ & $0 / 1$ & $0 / 0$ & $0 / 0$ & $0 / 0$ & $0 / 1$ \\
\hline \multicolumn{7}{|l|}{ Ptilodactylidae } \\
\hline Ptilodactyla sp. & $\mathrm{O}$ & $0 / 0$ & $0 / 0$ & $0 / 0$ & $2 / 4$ & $2 / 4$ \\
\hline
\end{tabular}


Table A1. Cont.

\begin{tabular}{|c|c|c|c|c|c|c|}
\hline Species & Group & Louisiana & Mississippi & North Carolina & Texas & Total \\
\hline \multicolumn{7}{|l|}{ Scarabaeidae } \\
\hline Anomala sp. & $\mathrm{O}$ & $0 / 0$ & $0 / 0$ & $0 / 0$ & $0 / 2$ & $0 / 2$ \\
\hline Ataenius imbricatus Melsheimer & $\mathrm{O}$ & $0 / 0$ & $0 / 1$ & $0 / 0$ & $0 / 0$ & $0 / 1$ \\
\hline Ataenius insculptus Horn & $\mathrm{O}$ & $0 / 0$ & $2 / 1$ & $0 / 0$ & $0 / 0$ & $2 / 1$ \\
\hline Ataenius sp. & $\mathrm{O}$ & $0 / 0$ & $1 / 0$ & $0 / 0$ & $0 / 0$ & $1 / 0$ \\
\hline Ateuchus histeroides Weber & $\mathrm{O}$ & $0 / 0$ & $0 / 0$ & $0 / 0$ & $2 / 1$ & $2 / 1$ \\
\hline Canthon ebenus Say & $\mathrm{O}$ & $0 / 0$ & $0 / 0$ & $0 / 0$ & $7 / 7$ & $7 / 7$ \\
\hline Canthon viridis (Beauvois) & $\mathrm{O}$ & $0 / 0$ & $0 / 1$ & $0 / 0$ & $1 / 1$ & $1 / 2$ \\
\hline Copris minutus (Drury) & $\mathrm{O}$ & $0 / 0$ & $1 / 0$ & $0 / 2$ & $0 / 0$ & $1 / 2$ \\
\hline Deltochilum gibbosum (Fabricius) & $\mathrm{O}$ & $0 / 0$ & $0 / 0$ & $3 / 0$ & $10 / 3$ & $13 / 3$ \\
\hline Digitonthophagus gazella (Fabricius) & $\mathrm{O}$ & $0 / 0$ & $1 / 0$ & $0 / 0$ & $0 / 0$ & $1 / 0$ \\
\hline Diplotaxis sp. & $\mathrm{O}$ & $0 / 0$ & $5 / 1$ & $0 / 0$ & $3 / 6$ & $8 / 7$ \\
\hline Euphoria sepulcralis (Fabricius) & $\mathrm{O}$ & $0 / 0$ & $0 / 0$ & $0 / 0$ & $2 / 7$ & $2 / 7$ \\
\hline Melanocanthon sp. & $\mathrm{O}$ & $0 / 0$ & $0 / 0$ & $0 / 0$ & $2 / 5$ & $2 / 5$ \\
\hline Onthophagus hecate (Panzer) & $\mathrm{O}$ & $0 / 0$ & $1 / 0$ & $0 / 0$ & $10 / 7$ & $11 / 7$ \\
\hline Onthophagus medorensis Brown & $\mathrm{O}$ & $0 / 0$ & $0 / 0$ & $0 / 0$ & $31 / 16$ & $31 / 16$ \\
\hline Onthophagus pennsylvanicus Harold & $\mathrm{O}$ & $0 / 0$ & $0 / 0$ & $0 / 0$ & $2 / 0$ & $2 / 0$ \\
\hline Onthophagus striatulus (Beauvois) & $\mathrm{O}$ & $0 / 1$ & $2 / 0$ & $0 / 0$ & $2 / 2$ & $4 / 3$ \\
\hline Onthophagus tuberculifrons Harold & $\mathrm{O}$ & $0 / 0$ & $0 / 0$ & $0 / 0$ & $0 / 2$ & $0 / 2$ \\
\hline Onthophagus sp. & $\mathrm{O}$ & $0 / 0$ & $0 / 0$ & $0 / 0$ & $0 / 1$ & $0 / 1$ \\
\hline Parataenius simulator (Harold) & $\mathrm{O}$ & $0 / 0$ & $0 / 0$ & $0 / 0$ & $0 / 1$ & $0 / 1$ \\
\hline Phyllophaga forsteri (Burmeister) & $\mathrm{O}$ & $0 / 0$ & $1 / 2$ & $0 / 0$ & $0 / 0$ & $1 / 2$ \\
\hline Phyllophaga prunina (LeConte) & $\mathrm{O}$ & $2 / 3$ & $0 / 0$ & $0 / 0$ & $0 / 3$ & $2 / 6$ \\
\hline Phyllophaga prununculina (Burmeister) & $\mathrm{O}$ & $0 / 0$ & $0 / 1$ & $0 / 0$ & $0 / 0$ & $0 / 1$ \\
\hline Phyllophaga scitula (Horn) & $\mathrm{O}$ & $0 / 0$ & $0 / 0$ & $0 / 0$ & $0 / 1$ & $0 / 1$ \\
\hline Phyllophaga sp. & $\mathrm{O}$ & $1 / 0$ & $0 / 0$ & $1 / 1$ & $0 / 0$ & $2 / 1$ \\
\hline Phyllophaga tristis complex & $\mathrm{O}$ & $0 / 0$ & $0 / 0$ & $0 / 0$ & $1 / 0$ & $1 / 0$ \\
\hline Platytomus longulus (Cartwright) & $\mathrm{O}$ & $1 / 0$ & $0 / 0$ & $0 / 0$ & $0 / 0$ & $1 / 0$ \\
\hline Serica sp. & $\mathrm{O}$ & $0 / 0$ & $0 / 0$ & $0 / 0$ & $2 / 0$ & $2 / 0$ \\
\hline \multicolumn{7}{|l|}{ Scraptiidae } \\
\hline Scraptia sp. & $\mathrm{O}$ & $0 / 0$ & $0 / 0$ & $1 / 0$ & $0 / 0$ & $1 / 0$ \\
\hline \multicolumn{7}{|l|}{ Silphidae } \\
\hline Necrophila americana (Linnaeus) & $\mathrm{O}$ & $0 / 0$ & $0 / 0$ & $0 / 0$ & $0 / 2$ & $0 / 2$ \\
\hline \multicolumn{7}{|l|}{ Silvanidae } \\
\hline Ahasverus advena (Waltl) & $\mathrm{S}$ & $0 / 0$ & $0 / 0$ & $0 / 0$ & $0 / 1$ & $0 / 1$ \\
\hline Ahasverus rectus (LeConte) & $\mathrm{S}$ & $1 / 12$ & $1 / 6$ & $1 / 0$ & $7 / 14$ & $10 / 32$ \\
\hline Cathartosilvanus imbellis (LeConte) & S & $0 / 0$ & $0 / 1$ & $0 / 0$ & $0 / 1$ & $0 / 2$ \\
\hline Silvanoprus scuticollis (Walker) & S & $0 / 0$ & $0 / 0$ & $0 / 0$ & $0 / 1$ & $0 / 1$ \\
\hline Silvanus muticus Sharp & S & $1 / 2$ & $0 / 0$ & $0 / 0$ & $2 / 6$ & $3 / 8$ \\
\hline \multicolumn{7}{|l|}{ Sphindidae } \\
\hline Sphindus sp. & $\mathrm{S}$ & $2 / 0$ & $1 / 0$ & $3 / 0$ & $1 / 4$ & $7 / 4$ \\
\hline \multicolumn{7}{|l|}{ Staphylinidae } \\
\hline Achenomorphus corticinus (Gravenhorst) & $\mathrm{O}$ & $1 / 0$ & $12 / 11$ & $0 / 4$ & $0 / 1$ & $13 / 16$ \\
\hline Anotylus sp. & $\mathrm{O}$ & $0 / 1$ & $0 / 0$ & $0 / 0$ & $27 / 39$ & $27 / 40$ \\
\hline Arthmius sp. & $\mathrm{O}$ & $0 / 0$ & $0 / 0$ & $1 / 0$ & $0 / 0$ & $1 / 0$ \\
\hline
\end{tabular}


Table A1. Cont.

\begin{tabular}{|c|c|c|c|c|c|c|}
\hline Species & Group & Louisiana & Mississippi & North Carolina & Texas & Total \\
\hline Astenus linearis (Erichson) & $\mathrm{O}$ & $0 / 1$ & $0 / 0$ & $0 / 0$ & $0 / 0$ & $0 / 1$ \\
\hline Astenus sp. & $\mathrm{O}$ & $1 / 2$ & $2 / 0$ & $0 / 0$ & $0 / 2$ & $3 / 4$ \\
\hline Baeocera laevis (Reitter) & $\mathrm{O}$ & $0 / 0$ & $0 / 0$ & $0 / 0$ & $1 / 0$ & $1 / 0$ \\
\hline Baeocera sp. & $\mathrm{O}$ & $0 / 0$ & $5 / 2$ & $0 / 1$ & $1 / 4$ & $6 / 7$ \\
\hline Belonuchus ephippiatus (Say) & $\mathrm{O}$ & $1 / 0$ & $0 / 1$ & $0 / 0$ & $1 / 0$ & $2 / 1$ \\
\hline Belonuchus rufipennis (Fabricius) & $\mathrm{S}$ & $0 / 0$ & $0 / 0$ & $1 / 0$ & $0 / 0$ & $1 / 0$ \\
\hline Bryoporus rufescens LeConte & $\mathrm{O}$ & $0 / 0$ & $3 / 1$ & $0 / 1$ & $0 / 0$ & $3 / 2$ \\
\hline Carpelimus sp. & $\mathrm{O}$ & $0 / 1$ & $0 / 0$ & $0 / 0$ & $0 / 1$ & $0 / 2$ \\
\hline Coproporus laevis LeConte & $\mathrm{S}$ & $0 / 0$ & $0 / 1$ & $0 / 0$ & $0 / 0$ & $0 / 1$ \\
\hline Coproporus ventriculus (Say) & $\mathrm{S}$ & $1 / 0$ & $0 / 0$ & $0 / 0$ & $0 / 0$ & $1 / 0$ \\
\hline Ctenisodes sp. & $\mathrm{O}$ & $0 / 0$ & $0 / 0$ & $0 / 0$ & $7 / 0$ & $7 / 0$ \\
\hline Decarthron sp. & $\mathrm{O}$ & $1 / 0$ & $0 / 0$ & $0 / 0$ & $0 / 0$ & $1 / 0$ \\
\hline Diochus schaumi Kraatz & $\mathrm{O}$ & $0 / 0$ & $0 / 0$ & $0 / 0$ & $0 / 1$ & $0 / 1$ \\
\hline Echiaster sp. & $\mathrm{O}$ & $0 / 0$ & $0 / 1$ & $0 / 0$ & $0 / 1$ & $0 / 2$ \\
\hline Erichsonius sp. & $\mathrm{O}$ & $0 / 1$ & $0 / 0$ & $0 / 0$ & $0 / 0$ & $0 / 1$ \\
\hline Euconnus sp. & $\mathrm{O}$ & $0 / 1$ & $0 / 0$ & $2 / 0$ & $0 / 1$ & $2 / 2$ \\
\hline Hesperus baltimorensis (Gravenhorst) & $\mathrm{O}$ & $0 / 2$ & $0 / 0$ & $2 / 0$ & $0 / 0$ & $2 / 2$ \\
\hline Homaeotarsus sp. & $\mathrm{O}$ & $0 / 0$ & $0 / 0$ & $0 / 0$ & $1 / 0$ & $1 / 0$ \\
\hline Hoplandria laevicollis (Notman) & $\mathrm{O}$ & $0 / 0$ & $0 / 0$ & $5 / 3$ & $0 / 0$ & $5 / 3$ \\
\hline Hoplandria sp. & $\mathrm{O}$ & $0 / 0$ & $0 / 1$ & $12 / 19$ & $1 / 0$ & $13 / 20$ \\
\hline Ischnosoma flavicolle (LeConte) & $\mathrm{O}$ & $1 / 0$ & $1 / 1$ & $0 / 0$ & $1 / 2$ & $3 / 3$ \\
\hline Myrmecocephalus concinnus (Erichson) & $\mathrm{S}$ & $0 / 2$ & $0 / 0$ & $0 / 0$ & $0 / 0$ & $0 / 2$ \\
\hline Oxybleptes davisi (Notman) & $\mathrm{O}$ & $37 / 4$ & $28 / 23$ & $0 / 0$ & $0 / 0$ & $65 / 27$ \\
\hline Oxypoda sp. & $\mathrm{O}$ & $0 / 0$ & $0 / 0$ & $0 / 0$ & $1 / 0$ & $1 / 0$ \\
\hline Palaminus sp. & $\mathrm{S}$ & $0 / 0$ & $1 / 2$ & $0 / 0$ & $2 / 0$ & $3 / 2$ \\
\hline Philonthus umbrinus (Gravenhorst) & $\mathrm{O}$ & $0 / 0$ & $0 / 0$ & $1 / 1$ & $0 / 0$ & $1 / 1$ \\
\hline Philotermes sp. & $\mathrm{S}$ & $0 / 0$ & $9 / 5$ & $0 / 0$ & $0 / 0$ & $9 / 5$ \\
\hline Pinophilus sp. & $\mathrm{O}$ & $1 / 0$ & $0 / 0$ & $0 / 0$ & $0 / 0$ & $1 / 0$ \\
\hline Platydracus fossator (Gravenhorst) & $\mathrm{O}$ & $0 / 0$ & $0 / 0$ & $1 / 0$ & $2 / 1$ & $3 / 1$ \\
\hline Platydracus sp. & $\mathrm{O}$ & $0 / 0$ & $0 / 0$ & $0 / 0$ & $2 / 1$ & $2 / 1$ \\
\hline Quedius capucinus (Gravenhorst) & $\mathrm{O}$ & $0 / 0$ & $0 / 0$ & $0 / 0$ & $0 / 1$ & $0 / 1$ \\
\hline Quedius verres Smetana & $\mathrm{O}$ & $0 / 0$ & $8 / 11$ & $0 / 0$ & $5 / 2$ & $13 / 13$ \\
\hline Rugilus sp. & $\mathrm{O}$ & $0 / 0$ & $3 / 0$ & $0 / 0$ & $2 / 9$ & $5 / 9$ \\
\hline Scaphisoma punctulatum LeConte & $\mathrm{O}$ & $0 / 0$ & $0 / 1$ & $0 / 0$ & $0 / 0$ & $0 / 1$ \\
\hline Scydmaenus sp. & $\mathrm{O}$ & $0 / 0$ & $0 / 0$ & $2 / 0$ & $0 / 0$ & $2 / 0$ \\
\hline Sepedophilus basalis (Erichson) & $\mathrm{O}$ & $0 / 0$ & $0 / 0$ & $0 / 0$ & $1 / 0$ & $1 / 0$ \\
\hline Sepedophilus crassus (Gravenhorst) & $\mathrm{O}$ & $0 / 0$ & $0 / 0$ & $0 / 1$ & $0 / 0$ & $0 / 1$ \\
\hline Sepedophilus debilis (Casey) & $\mathrm{O}$ & $0 / 0$ & $0 / 0$ & $1 / 0$ & $0 / 0$ & $1 / 0$ \\
\hline Sepedophilus sp. & $\mathrm{O}$ & $22 / 18$ & $2 / 2$ & $0 / 1$ & $13 / 19$ & $37 / 40$ \\
\hline Stenichnus sp. & $\mathrm{O}$ & $0 / 0$ & $0 / 0$ & $1 / 0$ & $0 / 0$ & $1 / 0$ \\
\hline Tachinus fimbriatus Gravenhorst & $\mathrm{O}$ & $0 / 0$ & $0 / 0$ & $2 / 0$ & $0 / 0$ & $2 / 0$ \\
\hline Thoracophorus costalis (Erichson) & $\mathrm{S}$ & $0 / 0$ & $0 / 0$ & $0 / 0$ & $30 / 26$ & $30 / 26$ \\
\hline Tmesiphorus costalis LeConte & $\mathrm{O}$ & $0 / 0$ & $0 / 1$ & $0 / 0$ & $0 / 0$ & $0 / 1$ \\
\hline
\end{tabular}


Table A1. Cont.

\begin{tabular}{|c|c|c|c|c|c|c|}
\hline Species & Group & Louisiana & Mississippi & North Carolina & Texas & Total \\
\hline \multicolumn{7}{|l|}{ Tenebrionidae } \\
\hline Alobates morio (Fabricius) & $\mathrm{S}$ & $0 / 0$ & $0 / 0$ & $0 / 0$ & $1 / 0$ & $1 / 0$ \\
\hline Alphitophagus bifasciatus (Say) & $\mathrm{S}$ & $0 / 2$ & $0 / 1$ & $0 / 0$ & $0 / 0$ & $0 / 3$ \\
\hline Anaedus brunneus (Ziegler) & $\mathrm{O}$ & $1 / 0$ & $0 / 1$ & $0 / 0$ & $0 / 0$ & $1 / 1$ \\
\hline Corticeus thoracicus (Melsheimer) & $\mathrm{S}$ & $0 / 2$ & $0 / 0$ & $0 / 0$ & $0 / 1$ & $0 / 3$ \\
\hline Gondwanocrypticus obsoletus (Say) & $\mathrm{S}$ & $1 / 0$ & $0 / 0$ & $0 / 0$ & $0 / 0$ & $1 / 0$ \\
\hline Helops cisteloides (Germar) & $\mathrm{S}$ & $0 / 0$ & $0 / 0$ & $0 / 0$ & $1 / 1$ & $1 / 1$ \\
\hline Helops sp. & $\mathrm{S}$ & $0 / 0$ & $0 / 0$ & $0 / 0$ & $1 / 0$ & $1 / 0$ \\
\hline Hymenorus sp. & $\mathrm{S}$ & $3 / 4$ & $1 / 2$ & $0 / 0$ & $3 / 5$ & $7 / 11$ \\
\hline Isomira sp. & $\mathrm{S}$ & $0 / 0$ & $0 / 0$ & $0 / 0$ & $0 / 2$ & $0 / 2$ \\
\hline Lobopoda erythrocnemis (Germar) & $\mathrm{S}$ & $3 / 3$ & $2 / 0$ & $0 / 0$ & $11 / 7$ & $16 / 10$ \\
\hline Opatrinus minimus (Beauvois) & $\mathrm{O}$ & $0 / 0$ & $0 / 0$ & $0 / 0$ & $1 / 1$ & $1 / 1$ \\
\hline Platydema micans Zimmerman & $\mathrm{S}$ & $2 / 1$ & $0 / 0$ & $0 / 0$ & $2 / 2$ & $4 / 3$ \\
\hline Platydema ruficolle Laporte and Brullé & $\mathrm{S}$ & $0 / 0$ & $0 / 1$ & $0 / 0$ & $2 / 1$ & $2 / 2$ \\
\hline Platydema ruficorne (Sturm) & $\mathrm{S}$ & $0 / 1$ & $0 / 1$ & $0 / 0$ & $0 / 0$ & $0 / 2$ \\
\hline Polypleurus perforatus (Germar) & $\mathrm{S}$ & $0 / 1$ & $1 / 0$ & $0 / 0$ & $4 / 2$ & $5 / 3$ \\
\hline Statira gagatina (Melsheimer) & $\mathrm{O}$ & $0 / 0$ & $0 / 1$ & $0 / 0$ & $0 / 0$ & $0 / 1$ \\
\hline Statira $\mathrm{sp.}$ & $\mathrm{O}$ & $0 / 0$ & $0 / 0$ & $0 / 0$ & $0 / 2$ & $0 / 2$ \\
\hline Uloma imberbis LeConte & $\mathrm{S}$ & $0 / 0$ & $0 / 0$ & $2 / 0$ & $0 / 1$ & $2 / 1$ \\
\hline Uloma punctulata LeConte & $\mathrm{S}$ & $0 / 6$ & $2 / 4$ & $0 / 0$ & $0 / 1$ & $2 / 11$ \\
\hline \multicolumn{7}{|l|}{ Tetratomidae } \\
\hline Eustrophopsis bicolor (Fabricius) & $\mathrm{S}$ & $1 / 1$ & $0 / 0$ & $0 / 0$ & $0 / 1$ & $1 / 2$ \\
\hline Eustrophus tomentosus Say & $\mathrm{S}$ & $0 / 0$ & $0 / 0$ & $0 / 0$ & $1 / 0$ & $1 / 0$ \\
\hline \multicolumn{7}{|l|}{ Throscidae } \\
\hline Aulonothroscus convergens (Horn) & $\mathrm{O}$ & $0 / 0$ & $0 / 4$ & $0 / 0$ & $0 / 0$ & $0 / 4$ \\
\hline \multicolumn{7}{|l|}{ Trogidae } \\
\hline Omorgus monachus Herbst & $\mathrm{O}$ & $0 / 0$ & $0 / 0$ & $0 / 0$ & $0 / 1$ & $0 / 1$ \\
\hline Trox spinulosus Robinson & $\mathrm{O}$ & $0 / 0$ & $0 / 0$ & $0 / 0$ & $2 / 0$ & $2 / 0$ \\
\hline Trox variolatus Melsheimer & $\mathrm{O}$ & $1 / 1$ & $0 / 0$ & $0 / 0$ & $1 / 1$ & $2 / 2$ \\
\hline \multicolumn{7}{|l|}{ Trogossitidae } \\
\hline Temnoscheila virescens (Fabricius) & $\mathrm{S}$ & $0 / 2$ & $0 / 0$ & $0 / 0$ & $1 / 0$ & $1 / 2$ \\
\hline \multicolumn{7}{|l|}{ Zopheridae } \\
\hline Bitoma quadriguttata (Say) & $\mathrm{S}$ & $0 / 0$ & $0 / 1$ & $0 / 0$ & $0 / 0$ & $0 / 1$ \\
\hline Colydium lineola Say & $\mathrm{S}$ & $0 / 0$ & $0 / 0$ & $0 / 0$ & $0 / 1$ & $0 / 1$ \\
\hline Colydium nigripenne LeConte & $\mathrm{S}$ & $0 / 3$ & $0 / 9$ & $0 / 0$ & $0 / 3$ & $0 / 15$ \\
\hline Endeitoma dentata (Horn) & $\mathrm{S}$ & $0 / 1$ & $1 / 0$ & $0 / 0$ & $0 / 0$ & $1 / 1$ \\
\hline Endeitoma $\mathrm{sp}$. & $\mathrm{S}$ & $0 / 1$ & $0 / 0$ & $0 / 0$ & $0 / 0$ & $0 / 1$ \\
\hline Hyporhagus punctulatus Thomson & $\mathrm{S}$ & $0 / 0$ & $0 / 0$ & $0 / 0$ & $0 / 1$ & $0 / 1$ \\
\hline Lasconotus pusillus LeConte & $\mathrm{S}$ & $0 / 0$ & $0 / 0$ & $0 / 0$ & $0 / 4$ & $0 / 4$ \\
\hline Microsicus parvulus (Guerin) & $\mathrm{S}$ & $0 / 0$ & $0 / 0$ & $0 / 0$ & $4 / 1$ & $4 / 1$ \\
\hline Pycnomerus haematodes (Fabricius) & $\mathrm{S}$ & $0 / 0$ & $0 / 0$ & $0 / 0$ & $0 / 1$ & $0 / 1$ \\
\hline Pycnomerus sulcicollis LeConte & $\mathrm{S}$ & $0 / 4$ & $2 / 9$ & $0 / 0$ & $0 / 1$ & $2 / 14$ \\
\hline
\end{tabular}


Table A1. Cont.

\begin{tabular}{|c|c|c|c|c|c|c|}
\hline Species & Group & Louisiana & Mississippi & North Carolina & Texas & Total \\
\hline Total Individuals (reference/wood addition) & & $\begin{array}{l}753 \\
(269 / 484)\end{array}$ & $\begin{array}{l}1016 \\
(406 / 610)\end{array}$ & $\begin{array}{l}1104 \\
(502 / 602)\end{array}$ & $\begin{array}{l}2299 \\
(997 / 1302)\end{array}$ & $\begin{array}{l}5172 \\
(2174 / 2998)\end{array}$ \\
\hline Total Species (reference/wood addition) & & $\begin{array}{l}140 \\
(79 / 112)\end{array}$ & $\begin{array}{l}162 \\
(105 / 125) \\
\end{array}$ & $\begin{array}{l}108 \\
(82 / 72) \\
\end{array}$ & $\begin{array}{l}227 \\
(147 / 180) \\
\end{array}$ & $\begin{array}{l}378 \\
(261 / 302) \\
\end{array}$ \\
\hline
\end{tabular}

\section{References}

1. Elton, C.S. The Pattern of Animal Communities; Methuen and Co. Ltd.: London, UK, 1966.

2. Siitonen, J. Forest management, coarse woody debris and saproxylic organisms: Fennoscandian boreal forests as an example. Ecol. Bull. 2001, 49, 11-41.

3. Grove, S.J. Saproxylic insect ecology and the sustainable management of forests. Ann. Rev. Ecol. Sys. 2002, 33,1-23.

4. Speight, M.C.D. Saproxylic Invertebrates and Their Conservation; Council of Europe: Strasbourg, France, 1989.

5. Andrew, N.; Rodgerson, L.; York, A. Frequent fuel-reduction burning: The role of logs and associated leaf litter in the conservation of ant biodiversity. Austral Ecol. 2000, 25, 99-107.

6. Evans, A.M.; Clinton, P.W.; Allen, R.B.; Frampton, C.M. The influence of logs on the spatial distribution of litter-dwelling invertebrates and forest floor processes in New Zealand forests. For. Ecol. Manag. 2003, 184, 251-262.

7. Jabin, M.; Mohr, D.; Kappes, H.; Topp, W. Influence of deadwood on density of soil macro-arthropods in a managed oak-beech forest. For. Ecol. Manag. 2004, 194, 61-69.

8. Topp, W.; Kappes, H.; Kulfan, J.; Zach, P. Litter-dwelling beetles in primeval forests of Central Europe: Does deadwood matter? J. Insect Conserv. 2006, 10, 229-239.

9. Topp, W.; Kappes, H.; Kulfan, J.; Zach, P. Distribution pattern of woodlice (Isopoda) and millipedes (Diplopoda) in four primeval forests of the western Carpathians (Central Slovakia). Soil Biol. Biochem. 2006, 38, 43-50.

10. Kappes, H. Influence of coarse woody debris on the gastropod community of a managed calcareous beech forest in western Europe. J. Molluscan Stud. 2005, 71, 85-91.

11. Kappes, H., Relations between forest management and slug assemblages (Gastropoda) of deciduous regrowth forests. For. Ecol. Manag. 2006, 237, 450-457.

12. Kappes, H.; Topp, W.; Zach, P.; Kulfan, J. Coarse woody debris, soil properties and snails (Mollusca: Gastropoda) in European primeval forests of different environmental conditions. Eur. J. Soil Biol. 2006, 42, 139-146.

13. Kappes, H.; Catalano, C.; Topp, W. Coarse woody debris ameliorates chemical and biotic soil parameters of acidified broad-leaved forests. Appl.Soil Ecol. 2007, 36, 190-198.

14. Jabin, M.; Topp, W.; Kulfan, J.; Zach, P. The distribution pattern of centipedes in four primeval forests of central Slovakia. Biodivers. Conserv. 2007, 16, 3437-3445.

15. Ulyshen, M.D.; Hanula, J.L. Litter-dwelling arthropod abundance peaks near coarse woody debris in loblolly pine forests of the southeastern United States. Fla. Entomol. 2009, 92, 163-164. 
16. Castro, A.; Wise, D.H. Influence of fallen coarse woody debris on the diversity and community structure of forest-floor spiders (Arachnida: Araneae). For. Ecol. Manag. 2010, 260, 2088-2101.

17. Ulyshen, M.D.; Klooster, W.S.; Barrington, W.T.; Herms, D.A. Impacts of emerald ash borer-induced tree mortality on leaf litter arthropods and exotic earthworms. Pedobiologia 2011, 54, 261-265.

18. Maser, C.; Trappe, J.M. The Seen and Unseen World of the Fallen Tree; General Technical Report PNW-164; USDA Forest Service, Pacific Northwest Forest and Range Experiment Station: Portland, OR, USA, 1984.

19. Amaranthus, M.P.; Parrish, D.S.; Perry, D.A. Decaying logs as moisture reservoirs after drought and wildfire in Stewardship of soil, air and water resources. In Proceedings of Watershed 89, Juneau, AK, USA, 21-23 March 1989; pp. 191-194.

20. Marra, J.L.; Edmonds, R.L. Effects of coarse woody debris and soil depth on the density and diversity of soil invertebrates on clearcut and forested sites on the Olympic Peninsula, Washington. Environ. Entomol. 1998, 27, 1111-1124.

21. Tilman, D.; Socolow, R.; Foley, J.A.; Hill, J.; Larson, E.; Lynd, L.; Pacala, S.; Reilly, J.; Searchinger, T.; Somerville, C.; Williams, R. Beneficial biofuels-The food, energy, and environment trilemma. Science 2009, 325, 270-271.

22. Landis, D.A.; Werling, B.P. Arthropods and biofuel production systems in North America. Insect Sci. 2010, 17, 220-236.

23. Bengtsson, J.; Persson, T.; Lundkvist, H. Long-term effects of logging residue addition and removal on macroarthropods and enchytraeids. J. Appl. Ecol. 1997, 34, 1014-1022.

24. Barton, P.S.; Manning, A.D.; Gibb, H.; Wood, J.T.; Lindenmayer, D.B.; Cunningham, S.A. Experimental reduction of native vertebrate grazing and addition of logs benefit beetle diversity at multiple scales. J. Appl. Ecol. 2011, 48, 943-951.

25. Gunnarsson, B.; Nittérus, K.; Wirdenäs, P. Effects of logging residue removal on ground-active beetles in temperate forests. For. Ecol. Manag. 2004, 201, 229-239.

26. Grove, S.J.; Hanula, J.L. Insect Biodiversity and Dead Wood: Proceedings of a Symposium for the 22nd International Congress of Entomology; General Technical Report SRS-93; USDA Forest Service, Southern Research Station: Asheville, NC, USA, 2006; pp. 57-66.

27. Ulyshen, M.D.; Hanula, J.L. Responses of arthropods to large-scale manipulations of dead wood in loblolly pine stands of the southeastern United States. Environ. Entomol. 2009, 38, 1005-1012.

28. Houseweart, M.W.; Jennings, D.T.; Rea, J.C. Large capacity pitfall trap. Entomol. News 1979, 90, 51-54.

29. Ulyshen, M.D.; Hanula, J.L. Patterns of saproxylic beetle succession in loblolly pine. Agr. For. Entomol. 2010, 12, 187-194.

30. Hamilton, W.D. Diversity of Insect Faunas. Symposia of the Royal Entomological Society of London; Mound, L.A., Waloff, N., Eds.; Blackwell Scientific: Oxford, UK, 1978; pp. 154-175.

31. Cárcamo, H.A.; Parkinson, D. Distribution of ground beetle assemblages (Coleoptera, Carabidae) around sour gas processing plants in western Canada. Pedobiologia 1999, 43, 160-173.

32. Pearce, J.L.; Venier, L.A.; McKee, J.; Pedlar, J.; McKenney, D. Influence of habitat and microhabitat on carabid (Coleoptera: Carabidae) assemblages in four stand types. Can. Entomol. 2003, 135, 337-357. 
33. Latty, E.F.; Werner, S.M.; Mladenoff, D.J.; Raffa, K.F.; Sickley, T.A. Response of ground beetles (Carabidae) assemblages to logging history in northern hardwood-hemlock forests. For. Ecol. Manag. 2006, 222, 335-347.

34. Nittérus, K.; Gunnarsson, Å.; Gunnarsson, B. Manipulated structural variability affects the habitat choice of two ground-living beetle species in a laboratory experiment. Entomol. Fenn. 2008, 19, 122-128.

35. Nittérus, K.; Gunnarsson, B. Effect of microhabitat complexity on the local distribution of arthropods in clear-cuts. Environ. Entomol. 2006, 35, 1324-1333.

(C) 2012 by the authors; licensee MDPI, Basel, Switzerland. This article is an open access article distributed under the terms and conditions of the Creative Commons Attribution license (http://creativecommons.org/licenses/by/3.0/). 